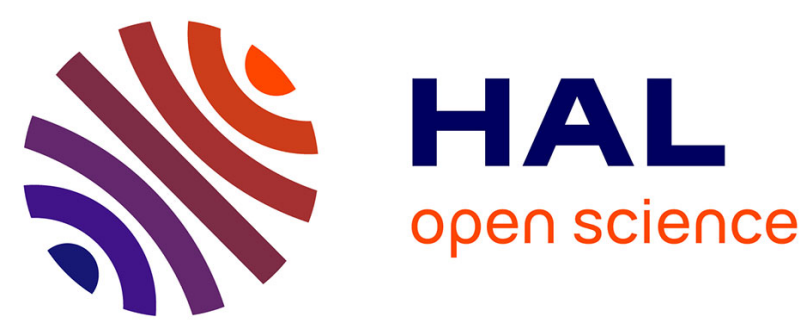

\title{
Circuit analog of a beam and its application to multimodal vibration damping, using piezoelectric transducers
}

Maurizio Porfiri, Francesco Dell'Isola, Fabio Massimo Frattale Mascioli

\section{To cite this version:}

Maurizio Porfiri, Francesco Dell'Isola, Fabio Massimo Frattale Mascioli. Circuit analog of a beam and its application to multimodal vibration damping, using piezoelectric transducers. International Journal of Circuit Theory and Applications, 2004, pp.32. hal-00499413

\section{HAL Id: hal-00499413 https://hal.science/hal-00499413}

Submitted on 15 Jul 2010

HAL is a multi-disciplinary open access archive for the deposit and dissemination of scientific research documents, whether they are published or not. The documents may come from teaching and research institutions in France or abroad, or from public or private research centers.
L'archive ouverte pluridisciplinaire HAL, est destinée au dépôt et à la diffusion de documents scientifiques de niveau recherche, publiés ou non, émanant des établissements d'enseignement et de recherche français ou étrangers, des laboratoires publics ou privés. 


\title{
Circuit analog of a beam and its application to multimodal vibration damping, using piezoelectric transducers
}

\author{
M. Porfiri ${ }^{1,2, \ddagger}$, F. dell’'Isola ${ }^{*, \dagger, 1}$ and F. M. Frattale Mascioli ${ }^{\S, 3}$ \\ ${ }^{1}$ Dipartimento di Ingegneria Strutturale e Geotecnica, Università di Roma 'La Sapienza', 00184 Roma, Italy \\ ${ }^{2}$ Engineering Science and Mechanics Department, Virginia Tech, Blacksburg VA 24061, U.S.A. \\ ${ }^{3}$ Dipartimento INFOCOM, Università di Roma 'La Sapienza', 00184 Roma, Italy
}

\begin{abstract}
SUMMARY
In this paper we solve the synthesis problem of finding a completely passive electric circuit ana$\log$ to a vibrating beam. The synthesis problem is of interest when one wants to suppress beam mechanical vibrations by using distributed piezoelectric transduction. Indeed, an effective electromechanical energy transduction is guaranteed when the electric circuit (interconnecting the transducers' terminals) is resonant at all mechanical resonance frequencies and is able to mimic all the mechanical modal shapes. The designed electric circuit behaves as an electric controller of mechanical vibrations (i.e. an electric vibration damper) once suitably endowed with a set of resistors. Because of its completely passive nature, it does not require external power units and stands as an economical means of controlling vibrations. Copyright (C) 2004 John Wiley \& Sons, Ltd.
\end{abstract}

KEY WORDS: passive electric networks; multiport synthesis; electric analogs; smart structures; multimodal vibrations; piezoelectric transducers; distributed control; passive damping

\section{INTRODUCTION: OVERVIEW OF VIBRATION SUPPRESSION STRATEGIES}

Extensive vibrations in mechanical systems can reduce the life of a structure and even contribute to its failure. However, by using piezoelectric transducers in conjunction with appropriate electric networks, the mechanical vibrational energy can be dissipated, strengthening the performance and extending the lifetime of the structure.

\footnotetext{
*Correspondence to: F. dell'Isola, Dipartimento di Ingegneria Strutturale e Geotecnica, Università di Roma, 'La Sapienza', Via Eudossiana 18, 00184 Roma, Italy.

†E-mail: francesco.dellisola@uniroma1.it

‡E-mail: maurizio.porfiri@uniroma1.it

§E-mail: mascioli@infocom.ing.uniroma1.it
}

Contract/grant sponsor: Engineering Science and Mechanics Department of the Virginia Polytechnic Institute and State University

Contract/grant sponsor: MIUR, Ministero per l'Innovazione, l'Università e la Ricerca Fondi Ricerca PRIN; contract/grant number: 2001097882_003

Contract/grant sponsor: Università di Roma 'La Sapienza' 
Piezoelectrics undergo deformation when an electric field is applied across their terminals (motor action, converse effect), and conversely produce voltage when strain is imposed (generator action, direct effect) (for a concise description of the piezoelectric effect see e.g. References [1,2]). Piezoelectricity was discovered by the Curie brothers (Pierre and Jacques Curie) in 1880, but it took several decades before this phenomenon could be exploited in real applications. It is reasonable to assess that the first use of piezoelectric materials dates back to the 1940s, during the second World War, as an ultrasonic detector for submarines. With the discovery of piezoceramics and the consequent development of sophisticated transducer architectures (for more details on the novel concepts used in the design of piezoelectric transducers see Reference [3]), the domain of the applications of piezoelectric materials has expanded considerably. Applications include space systems, aircraft, automotives, machine tools and medical systems (for more detailed information about new trends in the application of piezoelectric transducers for transportation vehicles, see e.g. Reference [4]). Extensive research efforts have been devoted to the active and passive vibrational damping of flexible structures, as undesired structural vibrations strongly affect structural performance, lifetime and reliability.

The so-called 'electronic damping' (see References [5-8]) was among the first applications conceived for the developed family of transducers in the field of active vibration control. A set of piezoelectric devices is placed on a host structure to sense and control the mechanical vibrations. The deformation of the sensing transducers results in electrical signals, which are conditioned by suitably designed feedback electronics and then applied to actuating transducers. The actuators convert the applied electrical energy into mechanical energy, transmitting mechanical control actions to the host structure. Such a concept proved to be effective, as the available actuators can exert forces of several hundred newtons as a response to voltage signals of several hundred volts, without losing their dielectric properties or undergoing destructive strain deformations. Indeed, the most remarkable feature of the modern piezoelectric transducers is their capability to remain in the linear range in the presence of strains on the order of $0.1 \%$ (see again Reference [3]). The common features of the control devices described in the framework of 'electronic damping' are represented by

- the differentiation of the sensing and the actuation systems;

- the localization of PZT actuators at limited, selected sites of the vibrating structure.

Both of these features are limits to controlling efficiency. The first one implies the need for a co-ordinating active system that controls the actuator action in response to the input from the sensors. The second feature implies an optimal localization problem (for both actuators and sensors), the solution of which depends on the particular mechanical vibration mode to be damped. The previously described vibration damping system is an example of an intelligent control system. This type of system has the ability to learn about its environment, process the information to reduce uncertainty, and plan, generate, and execute actions to either control or reduce to a minimum the undesired motion of all or some of its parts. It generally incorporates sensors, actuators, a controller, and a power supply unit. Most of the work in literature (see e.g. Reference [2]) has focused on active control, in which electric power is supplied to the actuators that exert actions on the host structure to suppress its vibrations. When active control systems are used, the piezoelectric transducers' driving requires complex power amplifiers and associated precise sensing electronics, resulting in the consumption of a significant amount of electrical power. Furthermore, the presence of an active controller can cause instability in 
the closed-loop system, the plant (vibrating structure) of which is naturally passive. Spillover phenomena can also be introduced, inducing dramatic oscillations of the structure at high frequencies.

An interesting development of 'electronic damping' has been proposed in References [9-12], where the design of optimal distributed electronic active controllers is addressed. The approach outlined in Reference [9] includes a distributed array of piezoelectric elements uniformly positioned over a host structure and a distributed interconnecting (active) electronic circuit. The piezoelectric layer is employed to measure (sense) the deformation of the flexible structure and to exert a continuous action at every point. The purpose of the distributed electronic circuit is to extract the complete state of the plant from the sensors, to optimally condition these signals, and to feed the actuators at high voltage. The resulting smart structure is able to efficiently suppress mechanical vibrations induced by broadband disturbance. Nevertheless, the intrinsic active nature of the controller and the complexity of the required circuitry may limit its technical feasibility and exploitation in industrial applications.

In Reference [13] the possibility of damping mechanical vibrations by means of a piezoelectric transducer positioned on a structural element and shunted with completely passive electrical circuits is investigated. In particular two different shunting circuits are considered: a resistive $(R)$ one and a resistive-inductive $(L R)$ one. By placing such an electrical impedance across the terminals of the piezoelectric transducer, the passive network is capable of damping structural vibrations. If a simple resistor is placed across the terminals of the transducer, the piezoelectric element will act as a viscoelastic damper. If the network consists of an inductorresistor circuit, the passive network combined with the inherent capacitance of the piezoelectric transducer creates damped electromechanical beating. The resonance can be tuned so that the piezoelectric element acts as a vibration absorber (parallelling the analysis of mechanical vibration absorbers exposed in Reference [14]). The method proposed in Reference [13] allows for an efficient single-mode control of structural vibrations whenever the resonant circuit is tuned to the mechanical mode to be suppressed. Nevertheless, the efficiency of the electromechanical coupling strongly depends on the position of the transducer over the host structure. Moreover, the technical feasibility of the passive piezoelectric controller proposed in Reference [13] is limited, since impossibly large inductances are required to produce low-frequency electrical resonance with the small inherent capacitance of the piezoelectric transducer. Many efforts have been devoted to simulating huge inductors by means of active electronic circuits. In particular, in Reference [15], an implementation method using a digital signal processor is presented, while in Reference [16] an analog realization exploiting operational amplifiers and multipliers is addressed. Nevertheless, when considerable structural vibrations are taken into account, several drawbacks can appear in these synthetic inductors due to saturation and non-linearities.

The principles of the 'piezoelectric shunting' technique proposed in Reference [13] can be applied to the multimodal control of vibrations (as shown in References $[17,18]$ ), by using intricate shunting impedances. Such a control methodology seems to present severe inconveniences: the used inductances are still very high and the damping efficiency, even for few modes, is reduced.

In order to overcome these drawbacks without compromising the advantages featured by passive control, in Reference [19] it is proposed to position on the host structure an array of piezoelectric elements and to interconnect the electric terminals of each pair of adjacent transducers via a floating $R L$ impedance. This strategy creates an archetype of what is known 
as a Piezo-ElectroMechanical structure. The main idea underlying this approach is to find how to get effective energy transduction from the mechanical to the electric form, as the electric form can be easily dissipated or stored. The main advantage of this strategy is the drastic reduction of employed optimal inductances, thus making conceivable the realization of a truly passive network. In this way one provides a synthetic support for low-speed electrical signals, to be effectively coupled to mechanical waves. (A precursory work to the aforementioned technique can be found in Reference [20].) Nevertheless, in this framework a multimodal control is not yet guaranteed and it is only possible to effectively damp one particular vibration mode.

\section{PROBLEM STATEMENT AND OBJECTIVE}

The optimization problem of finding the best distributed passive electric network (piezoelectrically coupled to the vibrating host structure) for achieving the most effective multimodal energy transduction has been addressed in Reference [21]. There, it is proven that in order to guarantee the maximum energy transfer between the mechanical and electric systems, they should be governed by the same partial differential equations. This property is often summarized by stating that the sought optimal distributed network should be the electric analog of the host structure. Therefore, the analog electrical circuit exhibits the same modal characteristics as those defining the host structure, so that a multiresonance electromechanical coupling can be established. Nevertheless, in Reference [21], no attention is paid to the suppression of structural vibrations. In Reference [22] the problem of finding an optimal distributed electric controller to attenuate propagating waves over any frequency range is tackled. The results presented in Reference [22] expand on those shown in Reference [21], assessing that the optimal continuous network for beam vibration damping should be governed by the same partial differential equations as the vibrating structure (i.e. elastica equation). But, at the same time, the network should be endowed with an internal dissipation proportional to the rate of change of the electric curvature (i.e. the second spatial derivative of electric potential).

The problem of synthesizing a completely passive lumped electrical circuit governed by a discrete approximation of the elastica equation has been extensively analysed in Reference [23]. Nevertheless, the proposed circuits have stern practical inconveniences, either due to negative inductors or multiport transformers. The former are typical active elements needing to be electronically simulated, and the latter are, in general, very heavy and their weights can represent a significant part of the mass of the overall smart structure. For a critical analysis of this control technique and comparisons among the electric controllers proposed in References $[19,23]$, see Reference [24].

In this paper we will find a completely passive lumped electric circuit analog to a vibrating beam, that consists only of inductors, capacitors and elementary two-port transformers. The proposed electric circuit will be synthesized following the subsequent design steps:

- finite difference discretization of the constitutive and balance equations for a vibrating Timoshenko beam,

- mobility representation of a beam element, 
- synthesis of a four-port grounded circuit (i.e. a four-port network containing only five terminals, one of which is a common ground terminal for all the ports), the admittance matrix of which parallels the beam element mobility (or mechanical impedance) matrix,

- cascade connection of the so-found networks to simulate the whole beam,

- neglection of beam shear deformability and rotatory inertia terms in the electric analog to achieve the Euler beam electric analog.

Once the beam electric analog has been designed, the problem of suppression of mechanically forced vibrations will be tackled. Therefore, an optimal multimodal electric dissipation of the mechanical energy will be achieved by placing suitable resistors on the found analog circuit.

\section{SYNTHESIS OF THE BEAM CIRCUIT ANALOG}

The governing equations for the vibrations of a Timoshenko beam are, see Reference [25]:

$$
\begin{aligned}
M^{\prime}+T & =\mathfrak{I} \dot{\Omega}, & \dot{M} & =K_{M} \Omega^{\prime} \\
T^{\prime} & =\rho \dot{V}, & \dot{T} & =K_{T}\left(V^{\prime}-\Omega\right)
\end{aligned}
$$

where $M$ indicates the bending moment, $T$ the shear contact action, $\mathfrak{I}$ the rotatory inertia, $V$ the deflection velocity, $\Omega$ the angular velocity of the cross-sections, $\rho$ the mass per unit length, $K_{T}$ the shear stiffness, $K_{M}$ the bending stiffness, and the superscripts dot and prime denote, respectively, time and space derivatives. $\|$

The first two partial differential equations on the left side of Equation (1) express the balance of the couple and shear contact actions, respectively, while the other two express the assumed linear constitutive behaviour.

In order to derive an analog circuit for the beam, let us non-dimensionalize the aforementioned governing equations introducing the scaling parameters $M_{0}, T_{0}, \tau_{0}, u_{0}, \vartheta_{0}$ and $l$. Hence, the dimensionless set of governing equations becomes:

$$
\begin{aligned}
\frac{M_{0}}{l} m^{\prime}+T_{0} t & =\frac{\vartheta_{0}}{\tau_{0}^{2}} \Im \dot{\omega}, \quad M_{0} \dot{m}=\frac{\vartheta_{0}}{l} K_{M} \omega^{\prime} \\
\frac{T_{0}}{l} t^{\prime} & =\frac{u_{0}}{\tau_{0}^{2}} \rho \dot{v}, \quad T_{0} \dot{t}=K_{T}\left(\frac{u_{0}}{l} v^{\prime}-\vartheta_{0} \omega\right)
\end{aligned}
$$

where each variable has been non-dimensionalized, and lower case letters indicate dimensionless quantities.**

Introducing a suitable finite differences approximation for the previous set of equations with respect to the space variable, it is straightforward to achieve the following set of first-order

\footnotetext{
T The reason why we are not interested in multiport ungrounded networks lies in the impossibility of guaranteeing that a certain pair of terminals behaves as a port when interconnected with another pair of terminals.

$\|$ Time will be denoted by $\tau$, while $x$ will denote the spatial abscissa.

** Since it does not introduce misunderstandings, dimensionless time and abscissa will be still denoted by $\tau$ and $x$ respectively.
} 
ordinary differential equations:

$$
\begin{aligned}
\frac{M_{0}}{l} \frac{m_{i+1}-m_{i}}{\delta}+T_{0} t_{i+1} & =\frac{\vartheta_{0}}{\tau_{0}^{2}} \Im \dot{\omega}_{i+1}, \quad M_{0} \dot{m}_{i}=\frac{\vartheta_{0}}{l} K_{M} \frac{\omega_{i+1}-\omega_{i}}{\delta} \\
\frac{T_{0}}{l} \frac{t_{i+1}-t_{i}}{\delta} & =\frac{u_{0}}{\tau_{0}^{2}} \rho \dot{v}_{i}, \quad T_{0} \dot{t}_{i+1}=K_{T}\left(\frac{u_{0}}{l} \frac{v_{i+1}-v_{i}}{\delta}-\vartheta_{0} \omega_{i+1}\right)
\end{aligned}
$$

where $\delta$ indicates the dimensionless spatial sampling step.

Let us explicitly remark that the adopted finite differences schemes alternate between the forward and the backward rule; this mixed approach will permit us to obtain symmetric higher order schemes when dealing with higher order governing equations expressed in terms of the kinematical descriptors, e.g. when determining the finite difference scheme for the elastica equations.

\subsection{Synthesis of the analog circuit via the voltage-velocity analogy}

Once a finite differences approximation for the mechanical impedance matrix of a beam element has been found, one well-established synthesis technique (see e.g. Reference [23]) requires paralleling the velocity with the voltage, and the contact actions with the currents. The velocities at the beam element ends are regarded as across variables and the contact actions as through variables.

The correspondence between the mechanical variables in Equation (3) and the electrical variables describing the analog network is:

$$
\begin{aligned}
& \left(V_{1}, I_{1}\right) \longleftrightarrow\left(\frac{V_{0} \tau_{0}}{\vartheta_{0}} \Omega_{i},-\frac{I_{0}}{M_{0}} M_{i}\right), \quad\left(V_{3}, I_{3}\right) \longleftrightarrow\left(\frac{V_{0} \tau_{0}}{\vartheta_{0}} \Omega_{i+1}, \frac{I_{0}}{M_{0}} M_{i+1}\right) \\
& \left(V_{2}, I_{2}\right) \longleftrightarrow\left(\frac{V_{0} \tau_{0}}{u_{0}} V_{i},-\frac{I_{0}}{T_{0}} T_{i}\right), \quad\left(V_{4}, I_{4}\right) \longleftrightarrow\left(\frac{V_{0} \tau_{0}}{u_{0}} V_{i+1}, \frac{I_{0}}{T_{0}} T_{i+1}\right)
\end{aligned}
$$

where $V_{0}$ and $I_{0}$ denote, respectively, a characteristic voltage and current.

By means of this analogy, the impedance matrix representation for the beam element parallels the admittance matrix representation for the analog four-port grounded network. The dimensionless mechanical impedance matrix of a beam element (see e.g. Reference [26]) is defined by:

$$
\left[\begin{array}{c}
-\widetilde{m}_{i} \\
-\widetilde{t}_{i} \\
\widetilde{m}_{i+1} \\
\widetilde{t}_{i+1}
\end{array}\right]=\mathbf{z}^{m}(\eta)\left[\begin{array}{c}
\widetilde{\omega}_{i} \\
\widetilde{v}_{i} \\
\widetilde{\omega}_{i+1} \\
\widetilde{v}_{i+1}
\end{array}\right]
$$

where $\widetilde{\bullet}$ denotes a one-sided Laplace transform, ${ }^{\dagger \dagger}$ and $\eta$ denotes the dimensionless Laplace variable. ${ }^{\ddagger \ddagger}$

\footnotetext{
${ }^{\dagger}$ Unless explicitly assumed, we set the initial conditions to zero.

¥† The dimensionless Laplace variable $\eta$ is related to the dimensional one $s$ by: $\eta=s \tau_{0}$.
} 
From Equations (3), one can immediately obtain:

$$
\mathbf{z}^{m}(\eta)=\left[\begin{array}{cccc}
\frac{1}{\eta} \frac{K_{M} \vartheta_{0}}{(\delta l) M_{0}} & 0 & -\frac{1}{\eta} \frac{K_{M} \vartheta_{0}}{(\delta l) M_{0}} & 0 \\
0 & \frac{1}{\eta} \frac{K_{T} u_{0}}{(\delta l) T_{0}}+\eta \frac{\rho u_{0}(\delta l)}{T_{0} \tau_{0}^{2}} & \frac{1}{\eta} \frac{K_{T} \vartheta_{0}}{T_{0}} & -\frac{1}{\eta} \frac{K_{T} u_{0}}{T_{0}(\delta l)} \\
-\frac{1}{\eta} \frac{K_{M} \vartheta_{0}}{(\delta l) M_{0}} & \frac{1}{\eta} \frac{K_{T} u_{0}}{M_{0}} & \frac{1}{\eta}\left(\frac{K_{M} \vartheta_{0}}{(\delta l) M_{0}}+\frac{K_{T} \vartheta_{0}(\delta l)}{M_{0}}\right)+\eta \frac{\Im \vartheta_{0}(\delta l)}{M_{0} \tau_{0}^{2}} & -\frac{1}{\eta} \frac{K_{T} u_{0}}{M_{0}} \\
0 & -\frac{1}{\eta} \frac{K_{T} u_{0}}{T_{0}(\delta l)} & -\frac{1}{\eta} \frac{K_{T} \vartheta_{0}}{T_{0}} & \frac{1}{\eta} \frac{K_{T} u_{0}}{(\delta l) T_{0}}
\end{array}\right]
$$

The dimensionless mechanical impedance matrix $\mathbf{z}^{m}(\eta)$ in Equation (5) can be decomposed in the Foster canonical form as follows, see Reference [27]:

$$
\mathbf{z}^{m}(\eta)=\frac{1}{\eta} \mathbf{z}_{0}^{m}+\eta \mathbf{z}_{\infty}^{m}
$$

with the residue matrices defined by:

$$
\begin{aligned}
& \mathbf{z}_{0}^{m}=\left[\begin{array}{cccc}
\frac{K_{M} \vartheta_{0}}{(\delta l) M_{0}} & 0 & -\frac{K_{M} \vartheta_{0}}{(\delta l) M_{0}} & 0 \\
0 & \frac{K_{T} u_{0}}{(\delta l) T_{0}} & \frac{K_{T} \vartheta_{0}}{T_{0}} & -\frac{K_{T} u_{0}}{T_{0}(\delta l)} \\
-\frac{K_{M} \vartheta_{0}}{(\delta l) M_{0}} & \frac{K_{T} u_{0}}{M_{0}} & \frac{K_{M} \vartheta_{0}}{(\delta l) M_{0}}+\frac{K_{T} \vartheta_{0}(\delta l)}{M_{0}} & -\frac{K_{T} u_{0}}{M_{0}} \\
0 & -\frac{K_{T} u_{0}}{T_{0}(\delta l)} & -\frac{K_{T} \vartheta_{0}}{T_{0}} & \frac{K_{T} u_{0}}{(\delta l) T_{0}}
\end{array}\right] \\
& \mathbf{z}_{\infty}^{m}=\left[\begin{array}{cccc}
0 & 0 & 0 & 0 \\
0 & \frac{\rho u_{0}(\delta l)}{T_{0} \tau_{0}^{2}} & 0 & 0 \\
0 & 0 & \frac{\Im \vartheta_{0}(\delta l)}{M_{0} \tau_{0}^{2}} & 0 \\
0 & 0 & 0 & 0
\end{array}\right]
\end{aligned}
$$

In order to synthesize an analog circuit for the entire transversely vibrating beam, it is sufficient to cascade connect a number of elementary analog networks of the beam element, thus assuring the compatibility of the displacement field and the equilibrium of the contact actions. 

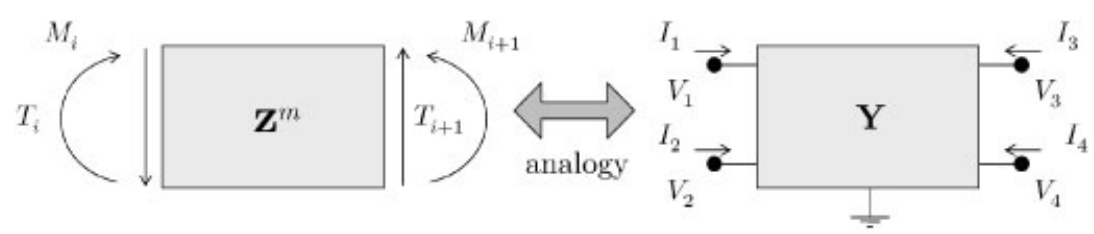

Figure 1. Analog circuit of a beam element utilizing the voltage-velocity analogy.

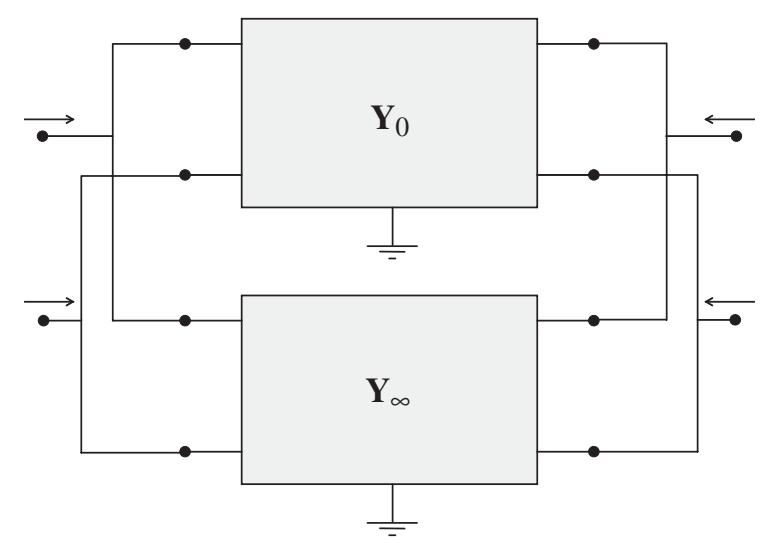

Figure 2. Realization of the analog circuit as the parallel connection of two elementary networks.

Therefore, a synthesis problem faced in this paper can be stated as:

\section{Problem 1}

Find a four port grounded network, the dimensionless admittance matrix of which is equal to the dimensionless impedance matrix $\mathbf{z}^{m}$, given in Equation (6).

Hence, we are looking for an electrical circuit (see Figure 1) whose admittance matrix $\mathbf{Y}(s)$ is

$$
\frac{1}{S} \frac{I_{0}}{V_{0} \tau_{0}} \mathbf{z}_{0}^{m}+s \frac{I_{0} \tau_{0}}{V_{0}} \mathbf{z}_{\infty}^{m}
$$

where $s$ represents the dimensional Laplace variable. The strategy developed to solve the addressed synthesis problem consists of the following steps:

1. synthesis of an inductive network whose admittance matrix $\mathbf{Y}_{0}(s)$ is equal to $1 / s\left(I_{0} / V_{0} \tau_{0}\right) \mathbf{z}_{0}^{m}$,

2. synthesis of a capacitive network whose admittance matrix $\mathbf{Y}_{\infty}(s)$ is equal to $s\left(I_{0} \tau_{0} / V_{0}\right) \mathbf{z}_{\infty}^{m}$,

3. parallel connection of the aforementioned electrical networks (see Figure 2) for the design of the circuit, the admittance matrix of which is $\mathbf{Y}(s)$.

In order to guarantee that the analog network is reciprocal, it is necessary to require the symmetry of the admittance matrix $\mathbf{Y}(s)$, which yields the following condition on the 


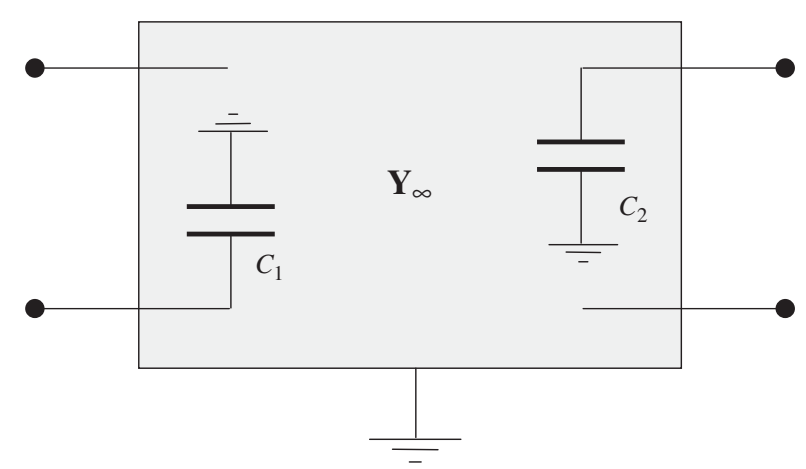

Figure 3. Realization of the network $\mathbf{Y}_{\infty}(s)$.

scaling parameters:

$$
u_{0} T_{0}=\vartheta_{0} M_{0}
$$

This condition, from a mechanical point of view, establishes that the characteristic work done by the bending moment $M_{0}$ on the rotation $\vartheta_{0}$, is equal to the characteristic work done by the shear contact action $T_{0}$ on the displacement $u_{0}$. Therefore, the scaling parameters cannot be chosen independently when one wants to design electric networks constituted only by reciprocal elements. Furthermore, introducing the parameters $\mu$ and $\kappa$, defined by:

$$
\mu=\frac{K_{T}}{K_{M}} \frac{u_{0}^{2}}{\vartheta_{0}^{2}}, \quad \kappa=\frac{K_{T}(\delta l)^{2}}{K_{M}}
$$

the residue at zero becomes:

$$
\mathbf{z}_{0}^{m}=\frac{K_{M} \vartheta_{0}}{(\delta l) M_{0}}\left[\begin{array}{cccc}
1 & 0 & -1 & 0 \\
0 & \mu & \sqrt{\mu \kappa} & -\mu \\
-1 & \sqrt{\mu \kappa} & 1+\kappa & -\sqrt{\mu \kappa} \\
0 & -\mu & -\sqrt{\mu \kappa} & \mu
\end{array}\right]
$$

The capacitive network can be designed as two capacitors connected at the second and third terminals of the grounded network as shown in Figure 3; the capacitance of these two elements are given by:

$$
C_{1}=\frac{I_{0}}{V_{0}} \frac{\rho u_{0}}{T_{0} \tau_{0}}(\delta l), \quad C_{2}=\frac{I_{0}}{V_{0}} \frac{I \vartheta_{0}}{M_{0} \tau_{0}}(\delta l)
$$

Therefore, the ratio of the two capacitances is given by:

$$
\frac{C_{1}}{C_{2}}=\frac{\rho}{\mathfrak{I}} \frac{u_{0}^{2}}{\vartheta_{0}^{2}}
$$


The design of the inductive circuit is much more involved, since the residue in zero is not diagonal. It is well known (see e.g. References [28, 29], regarding the synthesis of one-element type networks) that $\mathbf{Y}_{0}(s)$ is realizable as the admittance of an $n$-port network constituted only by inductors and containing only $n+1$ terminals, one of which is a common terminal for all the ports, if and only if the residue matrix $\mathbf{z}_{0}^{m}$ is dominant ${ }^{\S \S}$ and each of the off-diagonal terms is non-positive. $\uparrow \uparrow$ One can easily verify that $\mathbf{z}_{0}^{m}$ is not dominant and that some of the off-diagonal elements are positive. Therefore, even if the realization of $\mathbf{Y}_{0}(s)$ is not unique, it is impossible to synthesize it without using ideal transformers. In what follows, we will synthesize the considered network with a single two-port transformer. Towards this goal, we decompose $\mathbf{z}_{0}^{m}$ as the sum of the two following matrices:

$$
\mathbf{z}_{0}^{m}=\frac{K_{M} \vartheta_{0}}{(\delta l) M_{0}}\left[\begin{array}{cccc}
1 & 0 & -1 & 0 \\
0 & 0 & 0 & 0 \\
-1 & 0 & 1 & 0 \\
0 & 0 & 0 & 0
\end{array}\right]+\frac{K_{M} \vartheta_{0}}{(\delta l) M_{0}}\left[\begin{array}{cccc}
0 & 0 & 0 & 0 \\
0 & \mu & \sqrt{\mu \kappa} & -\mu \\
0 & \sqrt{\mu \kappa} & \kappa & -\sqrt{\mu \kappa} \\
0 & -\mu & -\sqrt{\mu \kappa} & \mu
\end{array}\right]
$$

By means of this decomposition, the synthesis problem has been drastically reduced to the design of a three-port grounded network, whose admittance matrix is:

$$
\mathbf{Y}_{0}^{\mathrm{red}}=\frac{1}{S} \frac{I_{0}}{V_{0} \tau_{0}} \frac{K_{M} \vartheta_{0}}{(\delta l) M_{0}}\left[\begin{array}{ccc}
\mu & \sqrt{\mu \kappa} & -\mu \\
\sqrt{\mu \kappa} & \kappa & -\sqrt{\mu \kappa} \\
-\mu & -\sqrt{\mu \kappa} & \mu
\end{array}\right]
$$

In fact, the first term on the RHS of Equation (8) can be immediately synthesized as an inductor interconnecting the first and the third terminals (see Figure 4). The value of the inductance is equal to:

$$
L_{1}=\frac{V_{0} \tau_{0} M_{0}}{I_{0} K_{M} \vartheta_{0}}(\delta l)
$$

Multiplying the inductance $L_{1}$ by the capacitance $C_{1}$ we get:

$$
C_{1} L_{1}=\frac{\rho}{K_{M}} \frac{u_{0}^{2}}{\vartheta_{0}^{2}}(\delta l)^{2}
$$

\footnotetext{
$\S \S \mathrm{A}$ real matrix is said to be dominant if each of its main-diagonal elements is not less than the sum of the absolute values of all the other elements in the same row.

ศ I If one is not restricting to $n+1$ terminals the following results are known: (i) a dominant matrix, with any distribution of signs in the off-diagonal terms, may always be realized with only inductors; (ii) paramountcy is a necessary (and sufficient, for the three-port case) condition for the matrix for its realizability without transformers.
} 


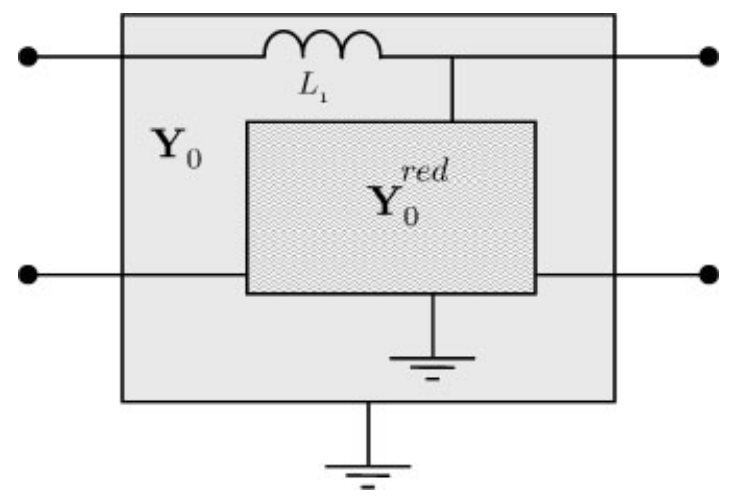

Figure 4. A first step towards the synthesis of $\mathbf{Y}_{0}(s)$.

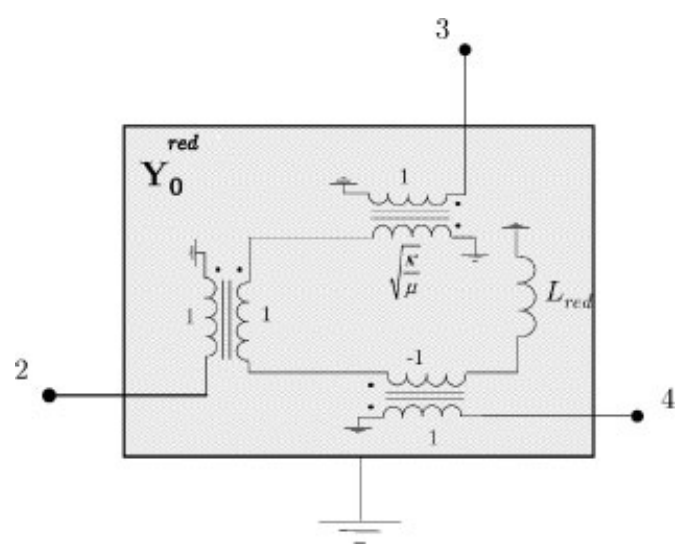

Figure 5. Direct design of $\mathbf{Y}_{0}(s)$.

The synthesis of a network governed by $\mathbf{Y}_{0}^{\text {red }}$ is still very tricky; nevertheless, one can immediately realize that the rank of $\mathbf{Y}_{0}^{\text {red }}$ is equal to one, hence it can be decomposed as:

$$
\mathbf{Y}_{0}^{\mathrm{red}}=\left[\begin{array}{c}
1 \\
\sqrt{\frac{\kappa}{\mu}} \\
-1
\end{array}\right]\left(\frac{1}{s} \frac{I_{0}}{V_{0} \tau_{0}} \frac{K_{M} \vartheta_{0}}{(\delta l) M_{0}} \mu\right)\left[1 \sqrt{\frac{\kappa}{\mu}}-1\right]
$$

and the circuit can be designed (see Reference [27]) as shown in Figure 5, with the inductance given by:

$$
L_{\mathrm{red}}=\frac{V_{0} \tau_{0} T_{0}}{I_{0} u_{0}} \frac{(\delta l)}{K_{T}}
$$




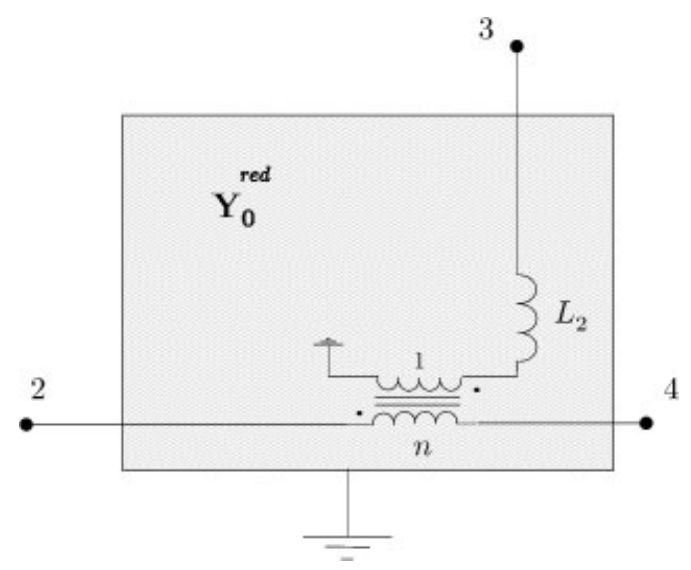

Figure 6. Minimal design of $\mathbf{Y}_{0}^{\text {red }}(s)$ (voltage-velocity analogy).

The previous topology can be further simplified by noticing that the turns-ratio of the first and third transformers are equal in absolute value and opposite in sign, as shown in Figure 6. In order to find the turns-ratio of the used transformer and the value of the introduced inductance, let us find the admittance matrix of the network shown in Figure 6 and compare it to $\mathbf{Y}_{0}^{\text {red }}$. The constitutive relation of the inductor $L_{2}$ and of the ideal transformer yield:

$$
\begin{aligned}
\tilde{I}_{2} & =-\tilde{I}_{4} \\
-\frac{\tilde{V} \tau_{2}-\tilde{V}_{4}}{n}+\tilde{V}_{3} & =s L_{2} \tilde{I}_{3} \\
n \tilde{I}_{4} & =\tilde{I}_{3}
\end{aligned}
$$

which establish the following admittance matrix:

$$
\frac{1}{s L_{2}}\left[\begin{array}{ccc}
1 / n^{2} & -1 / n & -1 / n^{2} \\
-1 / n & 1 & 1 / n \\
-1 / n^{2} & 1 / n & 1 / n^{2}
\end{array}\right]
$$

Comparing the aforementioned admittance matrix to $\mathbf{Y}_{0}^{\text {red }}$ :

$$
\left\{\begin{array} { l } 
{ \frac { 1 } { s L _ { 2 } } = \frac { 1 } { S } \frac { I _ { 0 } } { V _ { 0 } \tau _ { 0 } } \frac { K _ { M } \vartheta _ { 0 } } { ( \delta l ) M _ { 0 } } \kappa } \\
{ \frac { 1 } { n ^ { 2 } } = \frac { \mu } { \kappa } }
\end{array} \Rightarrow \left\{\begin{array}{l}
L_{2}=\frac{V_{0} \tau_{0}}{I_{0}} \frac{1}{K_{T}(\delta l)} \frac{M_{0}}{\vartheta_{0}} \\
n=\frac{\vartheta_{0}}{u_{0}}(\delta l)
\end{array}\right.\right.
$$

The inductance $L_{\mathrm{red}}$ is related to $L_{2}$ by:

$$
\frac{L_{2}}{L_{\mathrm{red}}}=\frac{1}{(\delta l)^{2}} \frac{u_{0}^{2}}{\vartheta_{0}^{2}}=\frac{1}{n^{2}}
$$


By inspection, one can immediately obtain the following set of relations between all the parameters so far introduced:

$$
n^{2} C_{1} L_{1}=\frac{\rho}{K_{M}}(\delta l)^{4}, \quad \frac{C_{1}}{C_{2}} n^{2}=\frac{\rho}{\mathfrak{I}}(\delta l)^{2}, \quad \frac{L_{1}}{L_{2}}=\frac{K_{T}}{K_{M}}(\delta l)^{2}
$$

The previous set of equations provides a group of conditions to be imposed on the employed circuit elements, completely independent of the arbitrarily chosen scaling parameters. Hence, it is easily seen that for every possible choice of scaling parameters there are always three fixed constraints on the circuit elements, which depend only on the physical properties of the beam and on the sampling step of the mesh.

In order to synthesize the analog circuit for the transversely-vibrating Timoshenko beam, it is sufficient to cascade connect a number of the found analog circuits for the generic beam element. Indeed, the electrical cascade connection corresponds exactly to the mechanical conditions of continuity of the contact actions and the kinematical descriptors over the length of the beam.

In what follows, we will neglect the shear deformation and the rotatory inertia: these hypotheses are justified when controlling and damping only low frequency vibrations. For the Euler beam model, thus obtained, the dimensionless governing equations (2) become:

$$
\begin{aligned}
\frac{M_{0}}{l} m^{\prime}+T_{0} t & =0, \quad M_{0} \dot{m}=\frac{\vartheta_{0}}{l} K_{M} \omega^{\prime} \\
\frac{T_{0}}{l} t^{\prime} & =\frac{u_{0}}{\tau_{0}^{2}} \rho \dot{v}, \quad \frac{u_{0}}{l} v^{\prime}=\vartheta_{0} \omega
\end{aligned}
$$

Deriving the first balance equation with respect to space and using the second balance equation, we get:

$$
\frac{M_{0}}{l} m^{\prime \prime}+T_{0}\left(\frac{l u_{0}}{T_{0} \tau_{0}^{2}} \rho \dot{v}\right)=0
$$

deriving the previous equation with respect to time and making use of the two constitutive relations in Equation (10) we finally get the elastica equation:

$$
\frac{K_{M} \tau_{0}^{2}}{\rho l^{4}} v^{I V}+\ddot{v}=0
$$

From Equation (9), by taking the limit as $K_{T} \rightarrow \infty$ and $\mathfrak{I} \rightarrow 0$ we obtain

$$
L_{2} \rightarrow 0, \quad C_{2} \rightarrow 0
$$

Therefore, for the so-called Euler beam, the analog circuit becomes the circuit depicted in Figure 7, with

$$
C_{1} L_{1} n^{2}=\frac{\rho}{K_{M}}(\delta l)^{4}
$$

The motion equation of the analog circuit sketched in Figure 7 in terms of the flux linkage $\Psi_{i}$ at the generic node $i$ (defined as the time integral of the voltage drop across the $i$ th 


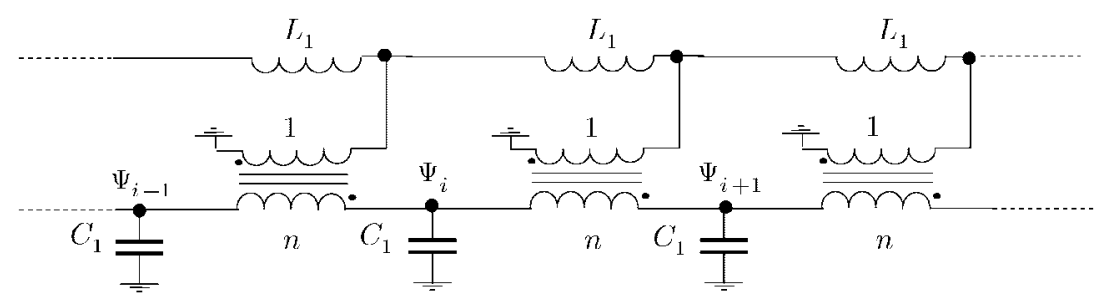

Figure 7. Circuit analog of an Euler beam corresponding to the voltage-velocity analogy.

capacitor) can be easily written as:

$$
\frac{\Psi_{i+2}-4 \Psi_{i+1}+6 \Psi_{i}-4 \Psi_{i-1}+\Psi_{i-2}}{L_{1} C_{1} n^{2}}+\ddot{\Psi}_{i}=0
$$

which represents a discrete form of the elastica, once condition (12) is satisfied.

In order to synthesize the analog circuit for the Euler beam using a finite difference approximation and exploiting the standard immittance matrices synthesis techniques, it is necessary to study initially the Timoshenko beam and then set the shear deformability and the rotatory inertia to zero. In fact, as the shear stiffness goes to infinity, the impedance matrix representation becomes impossible.

3.1.1. Constraints. The external constraints applied at the beam ends impose electrical constraints on the analog circuit (representing e.g. the electric terminations of the circuit depicted in Figure 7), which can be easily synthesized. The obtained boundary circuits are to be cascade connected to the ending modules of the analog network.

For instance, let us consider a simply supported beam, for which the mechanical conditions to be imposed are:

$$
\begin{array}{ll}
\widetilde{v}(0)=0, & \widetilde{m}(0)=0 \\
\widetilde{v}(1)=0, & \widetilde{m}(1)=0
\end{array}
$$

once the beam span has been chosen as the characteristic length for the non-dimensionalization. The electrical elements to simulate the free and the clamped end are easily found to be, respectively, determined by the following set of equations, once analogy (4) has been established:

$$
\begin{array}{cl}
\text { Left hinge: } & V_{2}=0, \quad I_{1}=0 \\
\text { Right hinge: } & V_{4}=0, \quad I_{3}=0
\end{array}
$$

\subsection{Synthesis of the analog circuit via the current-velocity analogy}

In the previous subsection, the synthesis of the analog network of a beam element has been performed exploiting the voltage--velocity analogy. In the following subsection, we will change our perspective and instead parallel the mechanical velocities with electric currents and mechanical contact actions with electric voltages. Therefore, contact actions will play the role of across variables, while velocities will be considered as through variables. The aforementioned 
assumptions are summarized by the following:

$$
\begin{aligned}
& \left(V_{1}, I_{1}\right) \longleftrightarrow\left(\frac{I_{0}}{M_{0}} M_{i},-\frac{V_{0} \tau_{0}}{\vartheta_{0}} \Omega_{i}\right), \quad\left(V_{3}, I_{3}\right) \longleftrightarrow\left(\frac{I_{0}}{M_{0}} M_{i+1}, \frac{V_{0} \tau_{0}}{\vartheta_{0}} \Omega_{i+1}\right) \\
& \left(V_{2}, I_{2}\right) \longleftrightarrow\left(\frac{I_{0}}{T_{0}} T_{i},-\frac{V_{0} \tau_{0}}{u_{0}} V_{i}\right), \quad\left(V_{4}, I_{4}\right) \longleftrightarrow\left(\frac{I_{0}}{T_{0}} T_{i+1}, \frac{V_{0} \tau_{0}}{u_{0}} V_{i+1}\right)
\end{aligned}
$$

In a similar way as what we have done in the previous subsection, let us define the dimensionless mobility matrix $\mathbf{m}(\eta)$ of the beam element relating the velocities at the two terminals to the contact actions:

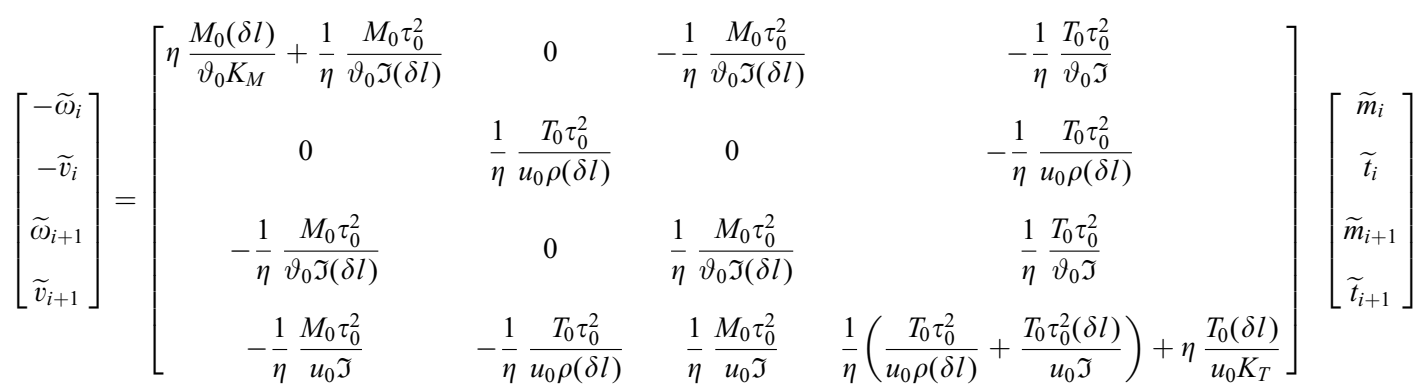

which admits the following Foster canonical form:

$$
\mathbf{m}(\eta)=\frac{1}{\eta} \mathbf{m}_{0}+\eta \mathbf{m}_{\infty}
$$

where the residue matrices are easily derived from Equation (5).

Thus the synthesis problem can be stated as:

\section{Problem 2}

Find a four-port grounded network, the dimensionless admittance matrix of which is equal to the dimensionless mobility matrix $\mathbf{m}$, given by Equation (14).

Hence, we are looking for an electrical circuit with admittance matrix $\mathbf{Y}(s)$

$$
\frac{1}{S} \frac{I_{0}}{V_{0} \tau_{0}} \mathbf{m}_{0}+s \frac{I_{0} \tau_{0}}{V_{0}} \mathbf{m}_{\infty}
$$

The solution of this problem is completely equivalent to the one addressed in the previous subsection, thus we will not go through all the synthesis details again, but we will only sketch the used procedure and duplicate the knowledge developed in the last section:

1. synthesis of an inductive network whose admittance matrix $\mathbf{Y}_{0}(s)$ is equal to $(1 / s)\left(I_{0} / V_{0} \tau_{0}\right) \mathbf{m}_{0}$

2. synthesis of a capacitive network whose admittance matrix $\mathbf{Y}_{\infty}(s)$ is equal to $s\left(I_{0} \tau_{0} / V_{0}\right) \mathbf{m}_{\infty}$, 


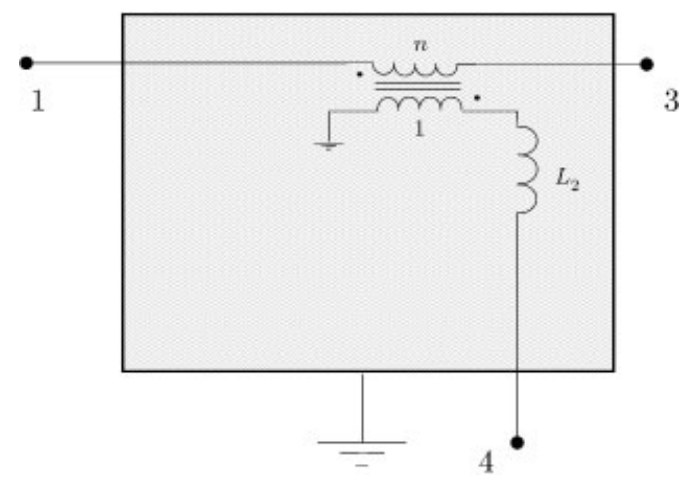

Figure 8. Minimal realization of $\mathbf{Y}_{0}^{\text {red }}(s)$ (current-velocity analogy).

3. parallel connection of the aforementioned electrical networks for the design of the circuit, the admittance matrix of which is $\mathbf{Y}(s)$.

In order to guarantee that the analog network is reciprocal, it is necessary to require the symmetry of the admittance matrix $\mathbf{Y}(s)$, which again yields Equation (7). The capacitive network can be designed as two uncoupled capacitors connected at the first and fourth terminals of the grounded network. The capacitances of these two elements are given by:

$$
C_{1}=\frac{I_{0} \tau_{0}}{V_{0}} \frac{M_{0}(\delta l)}{\vartheta_{0} K_{M}}, \quad C_{2}=\frac{I_{0} \tau_{0}}{V_{0}} \frac{T_{0}(\delta l)}{u_{0} K_{T}}
$$

The design of the inductive circuit is fully equivalent to the synthesis problem addressed in the previous section, since the matrix does not fulfil the necessary conditions for a realization without using ideal transformers. One may decompose the residue at zero as the sum of two matrices:

$$
\mathbf{m}_{0}=\mathbf{m}_{0}^{1}+\mathbf{m}_{0}^{2}
$$

where, $\mathbf{m}_{0}^{1}$ can be immediately synthesized as an inductor interconnecting the second and the fourth terminals, whose inductance is:

$$
L_{1}=\frac{V_{0}}{I_{0}} \frac{u_{0} \rho(\delta l)}{T_{0} \tau_{0}}
$$

and $\mathbf{m}_{0}^{2}$ can be synthesized by the circuit depicted in Figure 8 , where the inductance and the turns-ratio are given by:

$$
L_{2}=\frac{V_{0} u_{0}}{I_{0} T_{0} \tau_{0}} \frac{\mathfrak{I}}{(\delta l)}, \quad n=\frac{\vartheta_{0}}{u_{0}}(\delta l)
$$

Hence, the following relations between the circuit elements hold, independent of the used scaling parameters:

$$
\frac{C_{1}}{C_{2}} n^{2}=\frac{K_{T}}{K_{M}}(\delta l)^{2}, \quad C_{1} L_{1} n^{2}=\frac{\rho(\delta l)^{4}}{K_{M}}, \quad \frac{L_{1}}{L_{2}}=\frac{\rho}{\mathfrak{I}}(\delta l)^{2}
$$




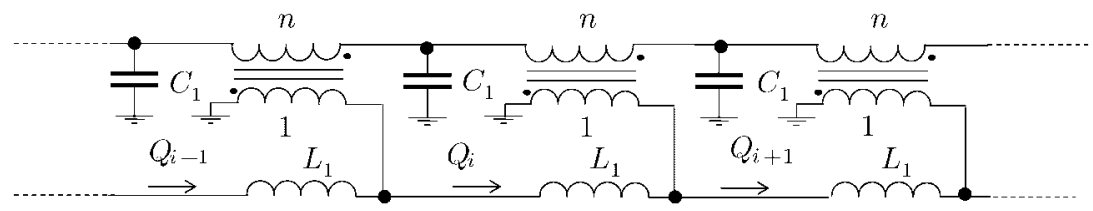

Figure 9. Circuit analog of an Euler beam corresponding to the current-velocity analogy.

When the Euler model is adopted, the inductance $L_{2}$ and the capacitance $C_{2}$ vanish, and the analog circuit in Figure 9 is obtained. The governing equation of such a circuit in terms of the time integral of the current flowing through the floating inductors, say $Q_{i}$, is:

$$
\frac{Q_{i+2}-4 Q_{i+1}+6 Q_{i}-4 Q_{i-1}+Q_{i-2}}{C_{1} n^{2}}+\ddot{Q}_{i} L_{1}=0
$$

3.2.1. Constraints. The circuit topology in Figures 7 and 9 are completely equivalent; nevertheless, the resulting analog circuits of a given constrained Euler beam are different. This difference lies in the boundary modules which, for the two proposed synthesis solutions, result in totally different circuital constraints. In fact, an electrical node which is short-circuited to ground for one solution results in an open-circuited terminal for the other, and viceversa.

\section{APPLICATION TO MULTIMODAL VIBRATION DAMPING}

The need for an electric analog of a vibrating beam arises when one wants to maximize the electromechanical energy transduction (see References [21,22]), dispensing with the use of an external power unit (i.e. in a completely passive way). In fact, as it will be shown in this section, the smart structure (say PiezoElectroMechanical beam, PEM for short) constituted by the host structure and an array of equally distributed piezoelectric elements interconnected as the capacitors of the analog circuits in Figures 7 and 9 (see for instance Figure 10) ) $^{\|\|}$allows a rapid and effective electromechanical energy transduction for every mechanical initial condition. This efficient energy transfer is assured by the internal resonance between the vibrating structure and the interconnecting network. A schematic plot of a simply supported PEM beam is presented in Figure 11, where the interconnecting network can be either of the presented analog circuits deprived of the capacitors.

In the previous section, the synthesis problem of finding a completely passive electric network analog to a beam has been solved, exploiting two different techniques: the former based on the voltage--velocity analogy, the latter on the current-velocity analogy. Both of the proposed solutions employ capacitors, inductors, and ideal two-port transformers.

In this section, a brief description of the two-fold nature (voltage driven actuator and strain sensor) of a piezoelectric transducer will be furnished. Then, the design of a PEM will be performed using both of the available circuit analogs from the previous section. The performances of the resulting controllers will be analysed and compared, stressing the need

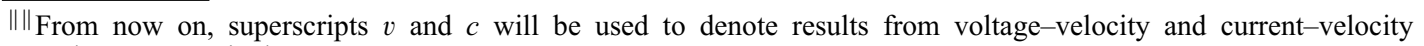
analogy, respectively. 


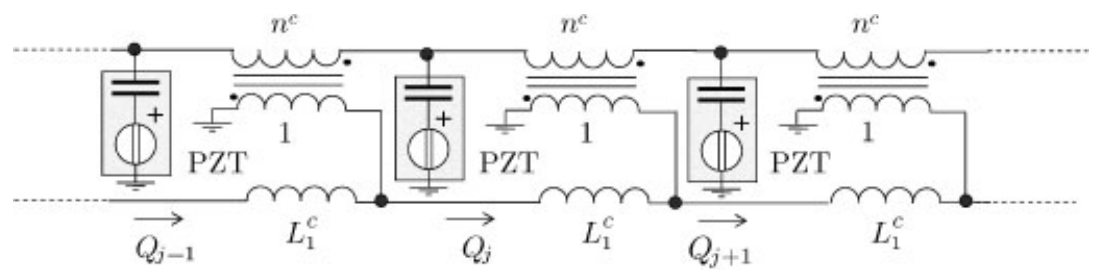

Figure 10. Sketch of the mechanically fed electric controller.

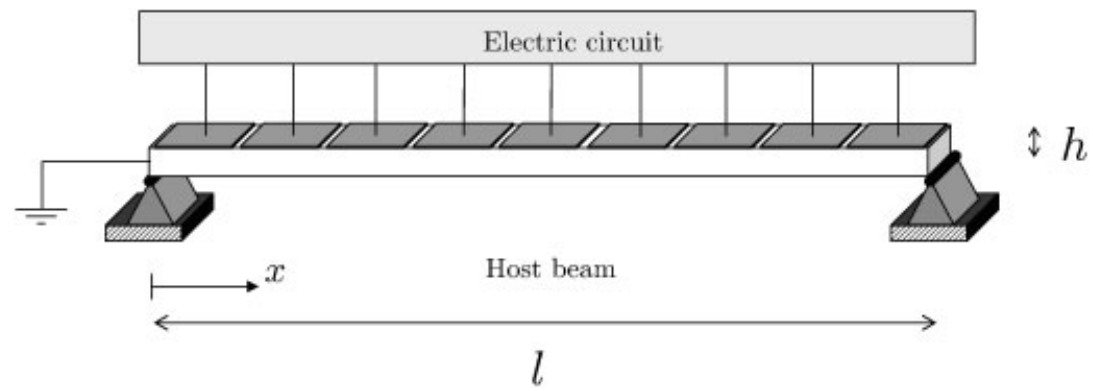

Figure 11. Schematics of a simply supported PEM beam.

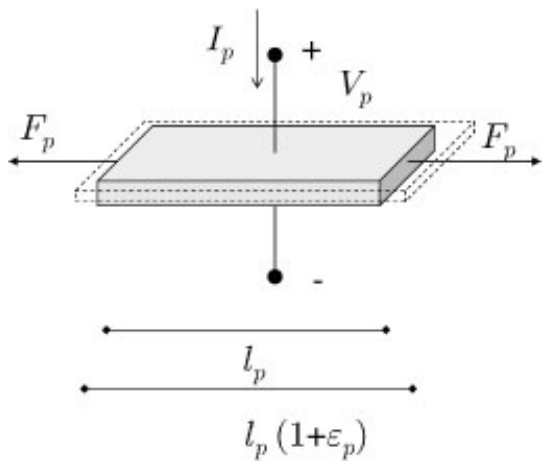

Figure 12. Sketch of a piezoelectric element.

for low inductances for a truly passive realization of the conceived device. Later, a modal analysis of the resulting structure will be provided for a better understanding of the conceived system and to assess its effectiveness when arbitrary initial conditions are prescribed. Finally, the synthesis of a dissipative electric network analog to a 'structurally damped beam' will be tackled to efficiently damp vibrations. 


\subsection{Basic model of a piezoelectric transducer}

An elementary model of a thin piezoelectric patch (see Figure 12) surface-bonded on a host beam, considers the transducer to be endowed with a two-fold behaviour:

- from an electrical point of view, it plays the role of a capacitor in parallel connection (series connection) with a current source (voltage source) driven by the mechanical time rate deformation;

- from a mechanical point of view, it behaves as a spring with two electrically driven pin forces applied at the patch ends.

Indeed, denoting the patch length with $l_{p}$ (assumed for the sake of simplicity to be equal to the grid step $\delta l$ ), the stored charge with $Q$, the exerted force with $F_{p}$, the strain with $\varepsilon_{p}$, and the applied voltage with $V_{p}$, the lumped constitutive equations can be written in terms of voltage and strain as (see e.g. References $[1,2]$ ):

$$
\begin{aligned}
& Q_{p}=k_{e e} V_{p}+k_{m e} \varepsilon_{p} l_{p} \\
& F_{p}=k_{m m} \varepsilon_{p} l_{p}-k_{m e} V_{p}
\end{aligned}
$$

or, in terms of charge and strain as:

$$
\begin{gathered}
V_{p}=\hat{k}_{e e} Q_{p}+\hat{k}_{m e} \varepsilon_{p} l_{p} \\
F_{p}=\hat{k}_{m m} \varepsilon_{p} l_{p}+\hat{k}_{m e} Q_{p}
\end{gathered}
$$

where the following relations between the constitutive parameters hold:

$$
\hat{k}_{e e}=\frac{1}{k_{e e}}, \quad \hat{k}_{m e}=-\frac{k_{m e}}{k_{e e}}, \quad \hat{k}_{m m}=\left(k_{m m}+\frac{k_{m e}^{2}}{k_{e e}}\right)
$$

From the first set of constitutive equations (15) the piezoelectric patch is recognized to electrically behave as a capacitor in parallel connection with a mechanically driven current source (Norton representation), while the second set (16) interprets the patch as a capacitor in series connection with a mechanically driven voltage source (Thevenin representation). The piezoelectric strain $\varepsilon_{p}$ is related to the mechanical deflection $U^{* * *}$ by the following no-slip condition:

$$
\varepsilon_{p}=\frac{h}{2} \overline{\left(U^{\prime \prime}\right)}
$$

where $h$ is the beam thickness (considering a rectangular cross section), and $\overline{\left(U^{\prime \prime}\right)}$ is the average curvature of the beam in the piezoelectrically-covered region. Since, the piezoelectric elements are adhesively bonded on the beam surface, their exerted forces $F_{p}$ induce bending moments applied at the patches ends.

\footnotetext{
*** The time derivative of $U$ is the velocity deflection $V$.
} 


\subsection{PEM beam models}

4.2.1. Voltage-velocity analogy. By substituting the surface-bonded piezoelectric transducers with the capacitors in Figure 7, and by adopting the constitutive relations (15), the following equations for the mechanically fed analog circuit are derived:

$$
\frac{\Psi_{i+2}-4 \Psi_{i+1}+6 \Psi_{i}-4 \Psi_{i-1}+\Psi_{i-2}}{L_{1}^{v}\left(n^{v}\right)^{2}}+k_{e e} \ddot{\Psi}_{i}+k_{m e} l_{p} \dot{\varepsilon}_{p_{i}}=0
$$

where $\varepsilon_{p_{i}}$ is the strain at the $i$ th patch and the superscript $v$ indicates voltage-velocity analogy.

When one can assume that the number of patches is sufficiently large, the following governing equation in terms of a continuously distributed flux linkage field $\Psi$ is obtained:

$$
\frac{1}{\lambda^{v}\left(v^{v}\right)^{2}} \Psi^{I V}+\gamma \ddot{\Psi}+c_{m e}^{v} \dot{U}^{\prime \prime}=0
$$

where the introduced parameters are given by:

$$
\gamma=\frac{k_{e e}}{(\delta l)}, \quad v^{v}=\frac{n^{v}}{(\delta l)}, \quad \lambda^{v}=\frac{L_{1}^{v}}{(\delta l)}, \quad c_{m e}^{v}=k_{m e} \frac{h}{2}
$$

Taking into account the additional stiffness and mass of the patches, the governing equation of the beam piezoelectrically covered is given by:

$$
\left(K_{M}+k_{m m}(\delta l)\left(\frac{h}{2}\right)^{2}\right) U^{I V}+\left(\rho+\rho_{p}\right) \ddot{U}-c_{m e}^{v} \dot{\Psi}^{\prime \prime}=0
$$

where $\rho_{p}$ denotes the mass per unit length of the piezoelectric covering layer.

Hence, the governing equations of the PEM beam are given by Equations (17) and (18). Introducing a characteristic displacement $U_{0}$, flux-linkage $\Psi_{0}$, and length and time $l$ and $\tau_{0}$, respectively, the governing equations become: ${ }^{\dagger \dagger}$

$$
\begin{aligned}
& \psi^{I V}+\alpha_{e}^{v} \ddot{\psi}+\beta_{e}^{v} \dot{u}^{\prime \prime}=0 \\
& u^{I V}+\alpha_{m}^{v} \ddot{u}-\beta_{m}^{v} \dot{\psi}^{\prime \prime}=0
\end{aligned}
$$

where the new parameters are given by:

$$
\begin{aligned}
& \alpha_{e}^{v}=\frac{l^{4} \lambda^{v}\left(v^{v}\right)^{2} \gamma}{\tau_{0}^{2}}, \quad \beta_{e}^{v}=\frac{l^{4} \lambda^{v}\left(v^{v}\right)^{2} c_{m e}^{v} U_{0}}{\psi_{0} \tau_{0} l^{2}} \\
& \alpha_{m}^{v}=\frac{l^{4}\left(\rho+\rho_{p}\right)}{\left(K_{M}+k_{m m}(\delta l)\left(\frac{h}{2}\right)^{2}\right) \tau_{0}^{2}}, \quad \beta_{m}^{v}=\frac{l^{4} c_{m e}^{v} \Psi_{0}}{u_{0}\left(K_{M}+k_{m m}(\delta l)\left(\frac{h}{2}\right)^{2}\right) \tau_{0} l^{2}}
\end{aligned}
$$

In order to guarantee an efficient multimodal control, it is necessary to tune the electrical circuit to the mechanical host beam (see Reference [21]) so that:

$$
\alpha_{m}^{v}=\alpha_{e}^{v}
$$

$\overline{\dagger_{\dagger} \text { As previously }}$ done, small letters will denote dimensionless quantities. 
which imposes the following relation on the circuit elements:

$$
\lambda^{v}\left(v^{v}\right)^{2} \gamma=\frac{\left(\rho+\rho_{p}\right)}{\left(K_{M}+k_{m m}(\delta l)(h / 2)^{2}\right)}
$$

Furthermore, to guarantee the desired efficient coupling ${ }^{\ddagger \ddagger}$ it is necessary to electrically parallel the mechanical boundary conditions. Let us underline that the two proposed electrical circuits yield different electrical boundary conditions with respect to the same mechanical ones.

It is very useful to choose the scaling quantities $U_{0}$ and $\Psi_{0}$ in order to obtain a gyroscopic coupling, namely:

$$
\beta_{e}^{v}=\beta_{m}^{v}
$$

which imposes the following relation:

$$
\frac{\Psi_{0}}{U_{0}}=\sqrt{\frac{\left(\rho+\rho_{p}\right)}{\gamma}} \Rightarrow \beta_{e}^{v} \equiv \beta_{m}^{v}=\frac{l^{2} c_{m e}^{v}}{\left(K_{M}+k_{m m}(\delta l)(h / 2)^{2}\right)} \sqrt{\frac{\left(\rho+\rho_{p}\right)}{\gamma}}
$$

4.2.2. Current-velocity analogy. The results shown in the present subsection stem directly from those derived in the previous discussion about the voltage-velocity analogy. Therefore, we will omit all the details and exhibit only the main equations.

By substituting the surface-bonded piezoelectric transducers with the capacitors in Figure 9, and by adopting the constitutive relations (16), the mechanically fed analog circuit in Figure 10 is found.

By assuming that the number of patches is sufficiently large, by introducing a characteristic length, time, displacement, and charge $\left(l, \tau_{0}, U_{0}, Q_{0}\right)$ satisfying

$$
\frac{Q_{0}}{U_{0}}=\sqrt{\frac{\left(\rho+\rho_{p}\right)}{\lambda^{c}}}
$$

and by tuning the electric system to the mechanical structure, i.e. by choosing

$$
\lambda^{c}\left(v^{c}\right)^{2} \gamma=\frac{\left(\rho+\rho_{p}\right)}{\left(K_{M}+\hat{k}_{m m}(\delta l)(h / 2)^{2}\right)}
$$

one obtains the following homogenized governing equations of the PEM beam featuring a symmetric elastic coupling:

$$
\begin{aligned}
& q^{I V}+\alpha^{c} \ddot{q}+\beta^{c} u^{I V}=0 \\
& u^{I V}+\alpha^{c} \ddot{u}+\beta^{c} q^{I V}=0
\end{aligned}
$$

\footnotetext{
$\overline{\ddagger \ddagger T h e ~ c o u p l i n g}$ effectiveness is essentially based on the same spectral properties of the electrical and mechanical systems.
} 
where the new parameters are given by:

$$
\begin{aligned}
\alpha^{c} & =\frac{l^{4}\left(\rho+\rho_{p}\right)}{\left(K_{M}+\hat{k}_{m m}(\delta l)(h / 2)^{2}\right) \tau_{0}^{2}} \\
\beta^{c} & =\frac{c_{m e}^{c}}{\left(K_{M}+\hat{k}_{m m}(\delta l)(h / 2)^{2}\right)} \sqrt{\frac{\left(\rho+\rho_{p}\right)}{\lambda^{c}}} \\
v^{c} & =\frac{n^{c}}{(\delta l)}, \quad \lambda^{c}=\frac{L_{1}^{c}}{(\delta l)}, \quad c_{m e}^{c}=\frac{1}{v^{c} \gamma} \frac{h}{2} k_{m e}
\end{aligned}
$$

and the superscript $c$ indicates the considered current-velocity analogy.

\subsection{Modal analysis of the current-velocity based PEM model}

The circuital connection in Figure 9 establishes a proportional elastic coupling between the electrical and mechanical system,,$\S \S$ while the connection in Figure 7 establishes a not proportional gyroscopic coupling. The proportional elastic coupling allows for the spillover suppression for any given set of mechanical boundary conditions, while the not proportional gyroscopic coupling leads, in general, to undesired spillover phenomena.

These circumstances suggest exploiting the current-velocity-based PEM model, instead of the voltage-velocity-based one, to control structural vibration for arbitrarily constrained beams.

Given the mechanical boundary conditions and then synthesizing the analog circuital connections, the modal shapes $\varphi_{i}^{0}(x)$ and frequency $\omega_{i}^{0}$ of the uncoupled electrical and mechanical systems coincide.

In order to determine the electromechanical modal shapes of the coupled system, the following eigenvalue problem has to be addressed:

$$
\left[\begin{array}{ll}
\frac{1}{\alpha^{c}}(\cdot)^{I V} & \frac{\beta^{c}}{\alpha^{c}}(\cdot)^{I V} \\
\frac{\beta^{c}}{\alpha^{c}}(\cdot)^{I V} & \frac{1}{\alpha^{c}}(\cdot)^{I V}
\end{array}\right]\left[\begin{array}{l}
q_{n} \\
u_{n}
\end{array}\right]=\omega_{n}^{2}\left[\begin{array}{l}
q_{n} \\
u_{n}
\end{array}\right]
$$

where both $q_{n}$ and $u_{n}$ should satisfy the prescribed boundary conditions.

We verify that the generic electromechanical eigenfunction is given by:

$$
\left[\begin{array}{l}
q_{n} \\
u_{n}
\end{array}\right]=\left[\begin{array}{l}
a_{n} \varphi_{n}^{0}(x) \\
b_{n} \varphi_{n}^{0}(x)
\end{array}\right]
$$

\footnotetext{
$\S \S \S$ By proportional (not proportional) coupling, we mean that the modal shapes of the mechanical structures are not modified (are modified) by the introduction of the electric network.
} 
Indeed, substituting Equation (23) in Equation (22) gives the following two-dimensional eigenvector problem:

$$
\left[\begin{array}{cc}
\left(\omega_{n}^{0}\right)^{2} & \beta^{c}\left(\omega_{n}^{0}\right)^{2} \\
\beta^{c}\left(\omega_{n}^{0}\right)^{2} & \left(\omega_{n}^{0}\right)^{2}
\end{array}\right]\left[\begin{array}{l}
a_{n} \\
b_{n}
\end{array}\right]=\omega_{n}^{2}\left[\begin{array}{l}
a_{n} \\
b_{n}
\end{array}\right]
$$

Hence, the coupled modal frequencies are determined as the roots of the characteristic polynomial

$$
P_{n}\left(\omega^{2}\right)=\left(\left(\omega_{n}^{0}\right)^{2}-\omega_{n}^{2}\right)^{2}-\left(\beta^{c}\left(\omega_{n}^{0}\right)^{2}\right)^{2}=\omega_{n}^{4}-2\left(\omega_{n}^{0}\right)^{2} \omega_{n}^{2}+\left(\omega_{n}^{0}\right)^{4}-\left(\beta^{c}\left(\omega_{n}^{0}\right)^{2}\right)^{2}
$$

i.e.:

$$
\omega_{n}^{2}=\left(\omega_{n}^{0}\right)^{2} \pm \sqrt{\left(\omega_{n}^{0}\right)^{4}-\left(\left(\omega_{n}^{0}\right)^{4}-\left(\beta^{c}\left(\omega_{n}^{0}\right)^{2}\right)^{2}\right)}=\left(\omega_{n}^{0}\right)^{2}\left(1 \pm \beta^{c}\right)
$$

The constants $a_{n}$ and $b_{n}$ can be evaluated as:

$$
\begin{aligned}
& \omega_{n}^{2}=\left(\omega_{n}^{0}\right)^{2}\left(1+\beta^{c}\right) \Rightarrow a_{n}=b_{n} \\
& \omega_{n}^{2}=\left(\omega_{n}^{0}\right)^{2}\left(1-\beta^{c}\right) \Rightarrow a_{n}=-b_{n}
\end{aligned}
$$

If the uncoupled modal shapes $\varphi_{n}^{0}$ are chosen to be normalized, in the sense that:

$$
\int_{0}^{1} \alpha^{c}\left(\varphi_{n}^{0}\right)^{2} \mathrm{~d} x=1
$$

then the absolute value of the constants $a_{n}$ and $b_{n}$ are chosen to be equal to $1 / \sqrt{2}$ to have normalized electromechanical eigenfunctions. Therefore the modal frequency and modal shapes of the PEM beam are:

$$
\left[\begin{array}{c}
q_{n}^{ \pm} \\
u_{n}^{ \pm}
\end{array}\right]=\left[\begin{array}{c}
1 / \sqrt{2} \varphi_{n}^{0}(x) \\
\pm 1 / \sqrt{2} \varphi_{n}^{0}(x)
\end{array}\right], \quad\left(\omega_{n}^{ \pm}\right)^{2}=\left(\omega_{n}^{0}\right)^{2}\left(1 \pm \beta^{c}\right)
$$

4.3.1. Free vibration of a PEM beam. When one wants to consider the free vibration of a PEM beam, it is useful to exploit the acquired knowledge of the modal shapes of the coupled systems (24). Expanding the solution in terms of the so-found eigenfunctions, we get:

$$
\left[\begin{array}{l}
q(x, \tau) \\
u(x, \tau)
\end{array}\right]=\sum_{n=1}^{\infty} c_{n}^{+}(\tau)\left[\begin{array}{l}
q_{n}^{+}(x) \\
u_{n}^{+}(x)
\end{array}\right]+\sum_{n=1}^{\infty} c_{n}^{-}(\tau)\left[\begin{array}{l}
q_{n}^{-}(x) \\
u_{n}^{-}(x)
\end{array}\right]
$$

The time evolution of the Fourier coefficients $c_{n}^{ \pm}(\tau)$ is determined by the diagonalized set of ordinary differential equations:

$$
\ddot{c}_{n}^{ \pm}(\tau)+\left(\omega_{n}^{ \pm}\right)^{2} c_{n}^{ \pm}(\tau)=0
$$


with initial conditions:

$$
\begin{aligned}
& c_{n}^{ \pm}(0)=\int_{0}^{1} \alpha^{c}\left[u(x, 0) u_{n}^{ \pm}(x)+q(x, 0) q_{n}^{ \pm}(x)\right] \mathrm{d} x \\
& \dot{c}_{n}^{ \pm}(0)=\int_{0}^{1} \alpha^{c}\left[\dot{u}(x, 0) u_{n}^{ \pm}(x)+\dot{q}(x, 0) q_{n}^{ \pm}(x)\right] \mathrm{d} x
\end{aligned}
$$

The solution of Equation (25) with the addressed initial conditions is:

$$
c_{n}^{ \pm}(\tau)=c_{n}^{ \pm}(0) \cos \left(\omega_{n}^{ \pm} \tau\right)+\frac{\dot{c}_{n}^{ \pm}(0)}{\omega_{n}^{ \pm}} \sin \left(\omega_{n}^{ \pm} \tau\right)
$$

When the initial conditions of the PEM beam consist of a sole deformation according the $k$ th mechanical modal shape $\varphi_{k}^{0}(x)$, the initial conditions of the Fourier coefficients $c_{n}^{ \pm}(\tau)$ become:

$$
\begin{aligned}
c_{n}^{ \pm}(0) & =0, \quad n \neq k \\
c_{k}^{+}(0) & =\frac{1}{\sqrt{2}} \\
c_{k}^{-}(0) & =-\frac{1}{\sqrt{2}} \\
\dot{c}_{n}^{ \pm}(0) & =0
\end{aligned}
$$

Thus, the time evolution of the PEM system becomes:

$$
\left[\begin{array}{l}
q(x, \tau) \\
u(x, \tau)
\end{array}\right]=\left[\begin{array}{c}
-\sin \left(\frac{\omega_{k}^{+}+\omega_{k}^{-}}{2} \tau\right) \sin \left(\frac{\omega_{k}^{+}-\omega_{k}^{-}}{2} \tau\right) \\
+\cos \left(\frac{\omega_{k}^{+}+\omega_{k}^{-}}{2} \tau\right) \cos \left(\frac{\omega_{k}^{+}-\omega_{k}^{-}}{2} \tau\right)
\end{array}\right] \varphi_{k}^{0}(x)
$$

which clearly emphasizes the absence of spillover phenomena and, generally speaking, proves that the distributed electric network, together with the piezoelectric transducers, provides a beating phenomenon in between the electrical and mechanical subsystems. Furthermore, let us stress that the ratio of the $k$ th carrier frequency to the $k$ th envelope frequency is independent of the mode number $k$.

\subsection{Damping structural vibrations}

Once an effective electromechanical energy transduction is established, it is conceivable to dissipate the beam energy into a properly inserted set of resistors. Therefore, this subsection is devoted to the design of an additional resistive network aimed towards multimodal vibration damping. 
The design procedure will be based on the following steps:

1. completion of Equation $(21)_{1}$ with a suitable electric dissipation contribution:

$$
\begin{array}{r}
q^{I V}+\alpha^{c} \ddot{q}+\beta^{c} u^{I V}+\mathbf{D}(\dot{q})=0 \\
u^{I V}+\alpha^{c} \ddot{u}-\beta^{c} q^{I V}=0
\end{array}
$$

D being a self-adjoint linear operator acting on the current field $\dot{q}$,

2. introduction of an applied load and statement of an objective functional representing the key features of the system steady state response,

3. minimization of the cost functional and consequent establishment of the optimal dissipation operator $\mathbf{D}$,

4. synthesis of a resistive lumped electrical network approximating the so-found operator.

To avoid undesired spillover phenomena in between different vibration modes, we will look for a dissipative electric network which does not modify the electromechanical eigenfunctions found in Section 4.3. Therefore, in the present paper the following restrictive assumption on D is made:

$$
\mathbf{D}=f\left((\cdot)^{I V}\right)
$$

$f$ being an analytic function which takes positive values on the real positive axis. बศा Thus, the dissipation operator is assumed to be the image of the fourth-order spatial derivative under a well-behaving operator-valued function (see e.g. Reference [30]). From Equation (28), by definition, it follows that:

$$
\mathbf{D}(\dot{q})=\sum_{n=1}^{\infty}\left(f\left(\alpha^{c}\left(\omega_{n}^{0}\right)^{2}\right)\left[\int_{0}^{1} \dot{q}(x, \tau) \alpha^{c} \varphi_{n}^{0}(x) \mathrm{d} x\right] \varphi_{n}^{0}(x)\right)
$$

Let us consider a generic applied load on the structure, represented by a forcing term $p(x, \tau)$ in Equation $(27)_{2}$ :

$$
\begin{aligned}
q^{I V}+\alpha^{c} \ddot{q}+\beta^{c} u^{I V}+\mathbf{D}(\dot{q}) & =0 \\
u^{I V}+\alpha^{c} \ddot{u}+\beta^{c} q^{I V} & =p
\end{aligned}
$$

Considering the modal expansions of $p, q$ and $u$ on $\varphi_{n}^{0}(x)$

$$
p(x, \tau)=\sum_{n=1}^{\infty} p_{n}(\tau) \varphi_{n}^{0}(x), \quad q(x, \tau)=\sum_{n=1}^{\infty} q_{n}(\tau) \varphi_{n}^{0}(x), \quad u(x, \tau)=\sum_{n=1}^{\infty} u_{n}(\tau) \varphi_{n}^{0}(x)
$$

and taking into account Equation (29), Equation (30) yields the following set of ordinary differential equations for the electrical and mechanical modal coefficients

$$
\begin{aligned}
\left(\omega_{n}^{0}\right)^{2} q_{n}+\ddot{q}_{n}+\beta^{c}\left(\omega_{n}^{0}\right)^{2} u_{n}+\frac{1}{\alpha^{c}} f\left(\alpha^{c}\left(\omega_{n}^{0}\right)^{2}\right) \dot{q}_{n} & =0 \\
\left(\omega_{n}^{0}\right)^{2} u_{n}+\ddot{u}_{n}+\beta^{c}\left(\omega_{n}^{0}\right)^{2} q_{n} & =p_{n}
\end{aligned}
$$

ฯฯ Tी The positiveness of $f(\lambda)$, when $\lambda$ is positive, assures the passivity of the electric network. 
Therefore, taking the Fourier transform of Equation (31) it is possible to introduce a transfer function relating the $n$th modal coefficient of the applied load to the mechanical evolution coefficient:

$$
H_{n}(\omega)=\frac{-\omega^{2}+\left(\omega_{n}^{0}\right)^{2}+\mathrm{i}\left(1 / \alpha^{c}\right) f\left(\alpha^{c}\left(\omega_{n}^{0}\right)^{2}\right) \omega}{\left(-\omega^{2}+\left(\omega_{n}^{0}\right)^{2}+\mathrm{i}\left(1 / \alpha^{c}\right) f\left(\alpha^{c}\left(\omega_{n}^{0}\right)^{2}\right) \omega\right)\left(-\omega^{2}+\left(\omega_{n}^{0}\right)^{2}\right)-\left(\beta^{c}\left(\omega_{n}^{0}\right)^{2}\right)^{2}}
$$

where $\mathrm{i}=\sqrt{-1}$.

The chosen optimality criterion requires the determination of the values of $f$ in order to minimize the $\mathscr{H}_{\infty}$ norm of the aforementioned transfer function, i.e. minimizing the supremum of $\left|H_{n}(\omega)\right|$ by appropriate choice of $f$. A particularly convenient approximate solution \|\|$\|$ to the considered optimization problem can be found by using the results in Reference [14]. We sketch here the main steps of the derivation. The first step of this process consists of finding the so-called 'invariant' frequencies $\omega_{n}^{S}$ and $\omega_{n}^{T}$ at which:

$$
\forall f_{1}, f_{2}:\left.\left|H_{n}\left(\omega_{n}^{S, T}\right)\right|_{f=f_{1}}|=| H_{n}\left(\omega_{n}^{S, T}\right)\right|_{f=f_{2}} \mid
$$

hence

$$
\omega_{n}^{S}=\sqrt{1-\frac{\beta^{c}}{\sqrt{2}}} \omega_{n}^{0}, \quad \omega_{n}^{T}=\sqrt{1+\frac{\beta^{c}}{\sqrt{2}}} \omega_{n}^{0}
$$

At these particular frequencies, since the internal resonance has already been established, it happens that:

$$
\forall \bar{f}:\left.\left|H_{n}\left(\omega_{n}^{S}\right)\right|_{f=\bar{f}}|=| H_{n}\left(\omega_{n}^{T}\right)\right|_{f=\bar{f}} \mid=\frac{\sqrt{2}}{\beta^{c}\left(\omega_{n}^{0}\right)^{2}}
$$

The second step consists of finding $f$ by enforcing that the amplitude of the transfer function at the resonance frequency $\omega_{n}^{0}$ equals the amplitude attained at the invariant frequencies, i.e.:

$$
\left|H_{n}\left(\omega_{n}^{0}\right)\right|=\left|H_{n}\left(\omega_{n}^{T}\right)\right| \equiv\left|H_{n}\left(\omega_{n}^{S}\right)\right|
$$

Thus, for the transfer function in Equation (32), we get:

$$
f\left(\alpha^{c}\left(\omega_{n}^{0}\right)^{2}\right)=\sqrt{2} \alpha^{c} \beta^{c} \omega_{n}^{0}
$$

Once the optimal expression for $f$ has been established, the dissipative operator is univocally determined by Equations (28) and (29) to be proportional to the positive square root of the fourth derivative operator, namely:

$$
\mathbf{D}=\sqrt{2} \sqrt{\alpha^{c}} \beta^{c} \sqrt{(\cdot)^{I V}}
$$

The mathematical properties of the square root of the fourth derivative operator have been extensively studied in References [31,32]. In Reference [31], such an operator is introduced to rigorously describe empirically observed damping rates in vibrating beams. While, in Reference [32], its mathematical properties are deeply analysed, and it is shown that this operator

||||| Although not technically optimal, the presented solution is very widespread due to its simplicity and appropriateness for experimental works. 


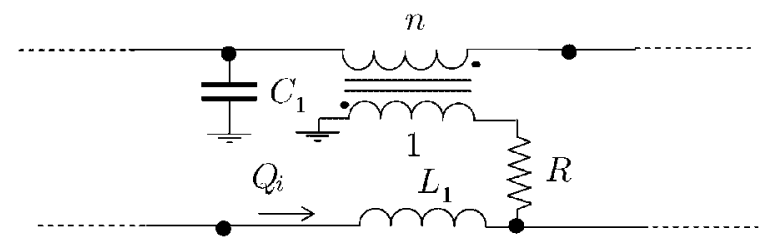

Figure 13. Analog circuit completed with optimally inserted resistors.

coincides with the negative second order derivative operator when special boundary conditions (called 'trigonometric', ${ }^{* * * *}$ see Reference [32]) are prescribed. Those boundary conditions are such to ensure purely trigonometric eigenfunctions for the fourth derivative operator. When such boundary conditions are met (for instance simply supporting) the dissipative operator is actually a differential operator and by exploiting finite difference approximations it is possible to synthesize a lumped approximating network. Otherwise, it is not a differential operator and its interpretation is rather obscure', [32]. Nevertheless, in these circumstances its approximation with the negative second order derivative seems to be reasonable for suboptimal solutions.

Restricting our attention to the so-called 'trigonometric cases', the finite difference approximation of $\mathbf{D}$ yields:

$$
\mathbf{D}(\dot{q}) \sim-\sqrt{2} \sqrt{\alpha^{c}} \beta^{c} \frac{\dot{q}_{i-1}-2 \dot{q}_{i}+\dot{q}_{i+1}}{\delta^{2}}
$$

which can be synthesized introducing a set of resistors as shown in Figure 13, where the resistance is:

$$
R=\frac{\sqrt{2}}{\delta l} \frac{c_{m e}^{c} \sqrt{\lambda^{c}}}{\sqrt{K_{M}+\hat{k}_{m m}(\delta l)(h / 2)^{2}}}
$$

By noticing that in Figure 13 the current flowing into the $i$ th transformer is $\dot{q}_{i}-\dot{q}_{i+1}$ (i.e. the port with unitary turns-ratio), it is easy to see that the resistor $R$ introducing a symmetric finite difference approximation of the second-order spatial derivative when computing the voltage across $L_{1}$. We explicitly remark that the found optimal circuital topology and resistance do not depend on the particular mode considered (i.e. its independent of $n$ ): as a consequence the synthesized circuit assures an effective multimodal damping.

\subsection{Design of a prototype}

In order to assess the engineering realizability and the efficiency of the proposed device, we will consider a simply supported aluminum beam, the geometry of which is presented in Table I.

Let us position ten transducers constituted by piezoceramic patches, produced by PiezoSystem, made of lead zirconate titanate [PZT]. The characteristics of these piezoceramic

\footnotetext{
****We refer to Reference [31] for the (rather involved) characterization of this class of boundary conditions. This is a delicate issue: we simply remark that the square root operator of the fourth-order derivative in the case of boundary conditions relative to a cantilever beam, is not a differential operator.
} 
Table I. Properties and dimensions of the host beam.

\begin{tabular}{lrc}
\hline Coefficient & Value & Units \\
\hline Length $(l)$ & 30 & $\mathrm{~cm}$ \\
Width $(w)$ & 2 & $\mathrm{~cm}$ \\
Height $(h)$ & 2 & $\mathrm{~mm}$ \\
Young's modulus $(E)$ & 70 & $\mathrm{GPa}$ \\
Mass density $\left(\rho^{V}\right)$ & 2700 & $\mathrm{~kg} / \mathrm{m}^{3}$ \\
\hline
\end{tabular}

Table II. Properties and dimensions of the piezoelectric transducers (Piezo System T110-H4E-602, made of PSI-5H4 piecoceramic).

\begin{tabular}{lcc}
\hline Coefficient & Value & Units \\
\hline Mass density $\left(\rho_{p}^{V}\right)$ & 7800 & $\mathrm{~kg} / \mathrm{m}^{3}$ \\
Elastic compliance $\left(s_{11}^{E}\right)$ & $1.612 \times 10^{-2}$ & $\mathrm{GPa}^{-1}$ \\
Piezoelectric constant $\left(d_{31}\right)$ & $-320 \times 10^{-12}$ & $\mathrm{~m} / \mathrm{V}$ \\
Dielectric constant $\left(\varepsilon_{3}^{T}\right)$ & $33.63 \times 10^{-9}$ & $\mathrm{~F} / \mathrm{m}$ \\
Thickness $\left(h_{p}\right)$ & 0.267 & $\mathrm{~mm}$ \\
Length $\left(l_{p}\right)$ & 30 & $\mathrm{~mm}$ \\
Width $\left(w_{p}\right)$ & 2.273 & $\mathrm{~mm}$ \\
\hline
\end{tabular}

Table III. Formulas for estimating key parameters of the PEM beam.

\begin{tabular}{lc}
\hline Coefficient & Formula \\
\hline Beam mass density $(\rho)$ & $\rho^{V} w h$ \\
Piezoelectric mass density $\left(\rho_{p}\right)$ & $\rho_{p}^{V} w_{p} \delta$ \\
Beam stiffness $\left(K_{M}\right)$ & $\frac{1}{12} E w h^{3}$ \\
Piezoelectric stiffness $\left(k_{m m}\right)$ & $\frac{h_{p} w_{p}}{s_{11}^{E} l_{p}}$ \\
Piezoelectric capacitance $\left(k_{e e}\right)$ & $\frac{\varepsilon_{3}^{T} w_{p} l_{p}}{h_{p}}\left(1-\frac{\left(d_{31}\right)^{2}}{\varepsilon_{3}^{T} s_{11}^{E}}\right)$ \\
Piezoelectric coupling $\left(k_{m e}\right)$ & $-d_{31} w_{p} s_{11}^{E}$ \\
\hline
\end{tabular}

transducers are listed in Table II. The relationships between the beam and piezoelectric material properties and the key parameters of the PEM are reported in Table III.

The capacitance and coupling coefficients of the transducers are:

$$
\begin{aligned}
k_{e e} & =61.3 \mathrm{nF} \\
\hat{k}_{m e} & =-3.576 \times 10^{6} \mathrm{~V} / \mathrm{m}
\end{aligned}
$$



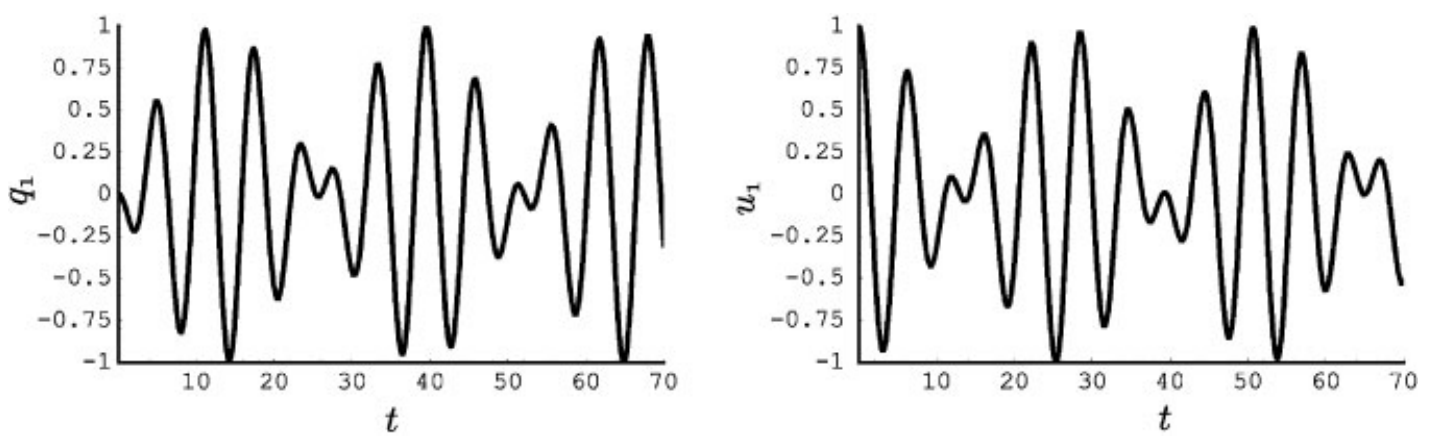

Figure 14. Time evolution of the first electrical and mechanical modal coefficients.

The stiffness and mass per unit length of the PEM beam can be evaluated as:

$$
\begin{aligned}
K_{M}+\hat{k}_{m m}(\delta l)\left(\frac{h}{2}\right)^{2} & =1.34 \mathrm{Nm}^{2} \\
\rho+\rho_{p} & =0.15 \mathrm{Kg} / \mathrm{m}
\end{aligned}
$$

In the following we will choose the angular frequency of the first mode as the frequency scaling parameter:

$$
\omega_{0}=\frac{\pi^{2}}{l^{2}} \sqrt{\frac{K_{M}+\hat{k}_{m m}(\delta l)(h / 2)^{2}}{\rho+\rho_{p}}}=328.3 \mathrm{rad} / \mathrm{s}
$$

Hence the dimensionless parameters can be numerically evaluated:

$$
\begin{aligned}
& \alpha^{c}=2.467 \\
& \beta^{c}=0.240
\end{aligned}
$$

From Equations (20) and (34) it is easy to calculate the following values for the optimal resistance and inductance in the interconnecting network:

$$
L_{1}^{c}=1.47 \mathrm{H}, \quad R=1661 \Omega
$$

assuming that the transformers turns-ratio are equal to one. In Figure 14 the time evolutions of the first electrical and mechanical modes, corresponding to a purely mechanical initial condition, are sketched, according to Equation (26). Furthermore Figures 15-17 show the frequency responses of the first three modes, when exploiting the optimal dissipative network presented in Figure 13.

\section{CONCLUSIONS}

Recent technological developments of piezoelectric transducers have made passive electric damping of structural vibrations realizable. Therefore, an interesting problem in the theory of circuits arises: how to electrically interconnect the piezoelectric transducers, placed on the 


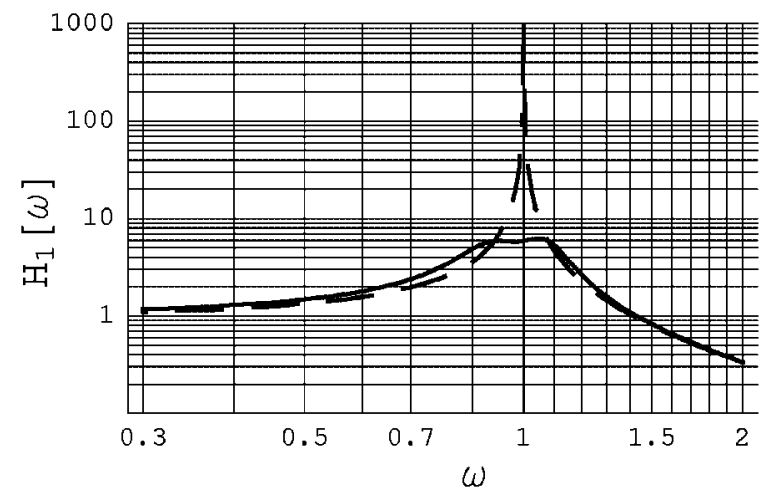

Figure 15. Frequency response around the first mode. The dashed curve represents the transfer function when all the piezoelectric elements are left open-circuited.

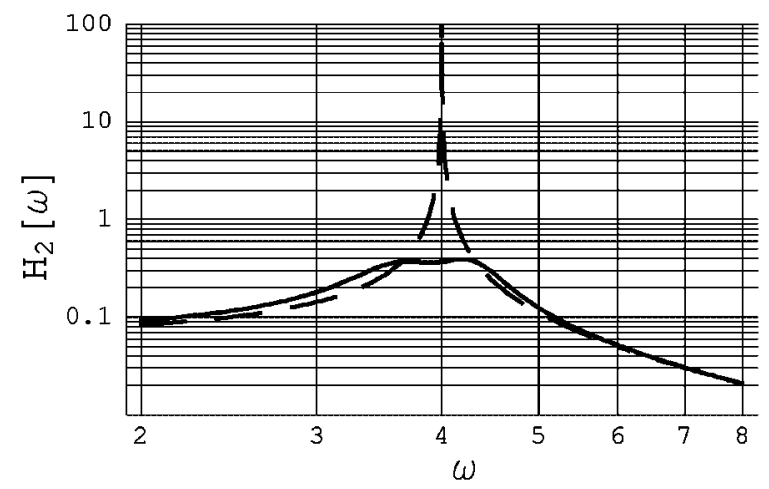

Figure 16. Frequency response around the second mode. The dashed curve represents the transfer function when all the piezoelectric elements are left open-circuited.

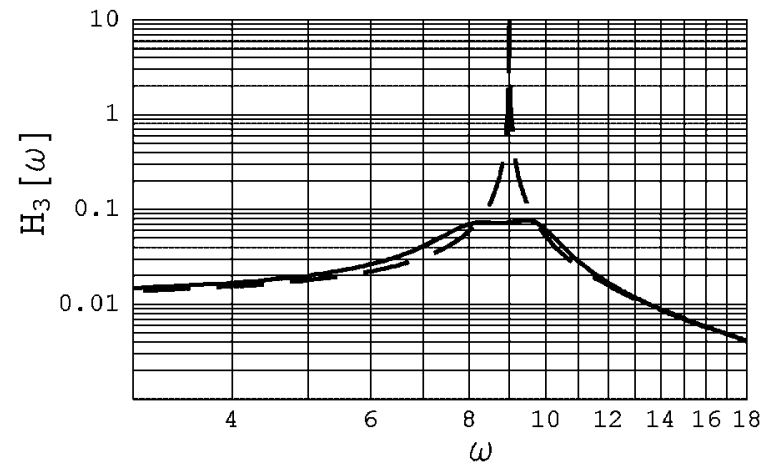

Figure 17. Frequency response around the third mode. The dashed curve represents the transfer function when all the piezoelectric elements are left open-circuited. 
structure, using a passive electric network in order to get an optimal damping of mechanical vibrations.

We refrain from studying optimal positioning problems: i.e. problems in which the actual placement of the transducers on the host structure is unknown. Instead, we assume that the array of distributed transducers is uniformly positioned on the structure and that the interconnecting network can be described by homogenized models. This class of smart structures has been studied in References [21,22], where the efficiency of analog circuits in obtaining effective electromechanical energy exchanges has been established.

In the present paper, the synthesis problem of finding a completely passive electric ana$\log$ of an Euler beam has been tackled. In Section 3, two different analogies are addressed and comparisons are drawn between the resulting circuits. The designed electric circuits are constituted only by capacitors, inductors, and ideal transformers; their hardware realization exploiting truly passive electric elements has been proved when designing a prototype.

The application of the found electric analogs to the multimodal vibrations damping is proposed in Section 4, where PEM beams are analysed.

Once an efficient coupling has been established, the completion of those lossless networks by suitable resistive networks (as shown in Section 4.4) guarantees a performing vibration attenuation without introducing spillover phenomena. The resulting smart structure is able to self-damp structural vibrations, dispensing with the use of any external power supply.

Future works will be devoted to the comparison between the performances of homogenized electric controllers and their lumped approximations and to the design of electric circuits approximating the square root of the fourth derivative differential operator when the prescribed boundary conditions are not 'trigonometric'.

\section{ACKNOWLEDGEMENTS}

The partial support of the Engineering Science and Mechanics Department of the Virginia Polytechnic Institute and State University is gratefully acknowledged by the authors. This presented research has been also partially supported by MIUR, Ministero per l'Innovazione, l'Università e la Ricerca Fondi Ricerca PRIN 'Sintesi di circuiti piezoelettrici e tecniche di disaccoppiamento per il controllo di vibrazioni meccaniche' (protocollo 2001097882_003) and by the Università di Roma 'La Sapienza'. The authors would also like to thank Prof. G. Martinelli for useful discussions about the problem and for a detailed review of this work and Ms A. White for her help in revising the English form of the paper. Finally, the authors would like to thank the reviewers for their comments that led to a considerable improvement in the manuscript.

\section{REFERENCES}

1. IEEE Std 176-1987 IEEE Standard on Piezoelectricity. The Institute of Electrical and Electronic Engineers: New York, 1987.

2. Chopra I. Review of state of art of smart structures and integrated systems. AIAA Journal 2002; 40(11): 2145-2187.

3. Niezreski C, Brei D, Balakrishnam S, Moskalik A. Piezoelectric actuation: state of the art. The Shock and Vibration Digest 2001; 33(4):269-280.

4. Boller C. State of the art and trends in using smart materials and systems in transportation vehicles. Proceedings of the Institute of Mechanical Engineers 1998; 212(Part 1):149-158.

5. Olsen HF. Electronic control of noise, vibration and reverberation. Journal of Acoustical Society 1956; 28: 976-972.

6. Forward RL, Swigert GJ. Electronic damping of orthogonal bending modes in a cylindrical mast-theory. Journal of Spacecraft and Rockets 1981; 18:5-10.

7. Hanagud S, Obal MW, Meyyappa M. Electronic damping techniques and active vibration control. Proceedings of the 26th AIAA/ASME/ASCE/AHS Structures, Structural Dynamics and Materials Conference, Orlando, Florida, USA, AIAA Paper 85-0752, April 1985. 
8. Hanagud S, Obal MW, Calise AJ. Optimal vibration control by the use of piezoceramic sensors and actuators. Journal of Guidance, Control and Dynamics 1992; 15(5):1199-1206.

9. Canon E, Lenczner M. Modelling of thin elastic plates with small piezoelectric inclusions and distributed electronic circuit. Models for inclusion that are small with respect to the thickness of the plate. Journal of Elasticity 1999; 55:111-141.

10. Hoffmann KH, Botkin ND. Homogenization of von Karman plates excited by piezoelectric patches. Zeitschrift fur Angewandte Mathematik und Mechanik 2000; 80(9):579-590.

11. Kader M, Lenczner M, Mrcarica Z. Distributed control based on distributed electronic circuits: application to vibration control. Microelectronics Reliability 2001; 41:1857-1866.

12. Bernadou M, Haenel C. Modelization and numerical analysis of active thin shell structures. Proceedings European Congress on Computational Methods in Applied Sciences and Engineering ECCOMAS 2000, Barcelona, 2000.

13. Hagood NW, von Flotow AH. 1991. Damping of structural vibrations with piezoelectric materials and passive electrical networks. Journal of Sound and Vibration 1991; 146(2):243-368.

14. Den Hartog J. Mechanical Vibrations. McGraw-Hill: New York, 1934.

15. Fleming AJ, Behrens S, Moheimani SOR. Synthetic impedance for implementation of piezoelectric shuntdamping circuits. Electronic Letters 2000; 36:1525-1526.

16. Keun-Ho R, In L. Adaptive shunting for vibration control of frequency-varying structures. Journal of Guidance, Control, and Dynamics 2001; 24(6):1223-1225.

17. Hollkamp JJ. Multimodal passive vibration suppression with piezoelectric materials and resonant shunts. Journal of Intelligent Material Systems and Structures 1994; 5:49-57.

18. Fleming AJ, Behrens S, Moheimani SOR. Optimization and implementation of multimode piezoelectric shunt damping systems. IEEE/ASME Transactions on Mechatronics 2002; 7(1):87-94.

19. dell'Isola F, Vidoli S. Continuum modelling of piezo-electromechanical truss beams: an application to vibration damping. Archive of Applied Mechanics 1998; 68:1-19.

20. Valis T, von Flotow AH, Hagood NW. An acousto-electromagnetic piezoelectric waveguide-couple. Active Materials and Adaptive Structures, Proceedings of the ADPA/AIAA/ASME/SPIE Conference November 1991 Alexandria Virginia Gareth J Knowles (ed.) Institution of Physics Publishing: Bristol, Philadelphia, 1991; 383-394.

21. Vidoli S, dell'Isola F. Modal coupling in one-dimensional electromechanical structured continua. Acta Mechanica 2000; 141:37-50.

22. dell'Isola F, Henneke EG, Porfiri M. Piezoelectromechanical structures: a survey of basic concepts and methodologies. Proceedings of the SPIE Smart Structures and Materials, Smart Structures and Integrated Systems, San Diego, vol. 5052, 2003; 392-402.

23. Alessandroni S, dell'Isola F, Porfiri M. A revival of electric analogs for vibrating mechanical systems aimed to their efficient control by PZT actuators. International Journal of Solids and Structures 2002; 39(20):5295-5324.

24. Maurini C, dell'Isola F, Del Vescovo D. Comparison of piezoelectronic networks acting as distributed vibration absorbers. Mechanical Systems and Signal Processing 2004; 18(5):1243-1271.

25. Soedel W. Vibrations of Shells and Plates (2nd edn). Marcel Dekker: New York, 1993.

26. Molloy CT. Four pole parameters in vibration analysis in mechanical impedance methods for mechanical vibrations. Colloquium on Mechanical Impedance Methods for Mechanical Vibrations presented at the ASME Annual Meeting 1958, New York, NY, 2 December (sponsored by Shock and Vibrating Committee Applied Mechanics Division, The American Society of Mechanical Engineers, New York, R. Plunkett (ed.)).

27. Newcomb RW. Linear Multiport Synthesis. McGraw-Hill: New York, 1966.

28. Slepian P, Weinbeg L. Synthesis. Synthesis applications of paramount and dominant matrices. Proceedings of the National Electronics Conference 1958; 14:611-630.

29. Panel. The realization of $n$-port networks without transformers: a panel discussion. Transactions on IRE Circuit Theory 1962; CT-9(3):202-214.

30. Pedersen M. Functional Analysis in Applied Mathematics and Engineering. Chapman \& Hall: Boca Raton, 1999.

31. Chen G, Russell DL. A mathematical model for linear elastic systems with structural damping. Quarterly of Applied Mathematics 1982; 39:433-454.

32. Russell DL. On the positive square root of the fourth derivative operator. Quarterly of Applied Mathematics 1988; 46:751-773.

33. Tang J, Wang KW. Active-passive hybrid piezoelectric networks for vibration control: comparisons and improvement. Smart Materials and Structures 2001; 10:794-806.

34. Miller DW, Crawley EF. Theoretical and experimental investigation of space-realizable inertial actuation for passive and active structural control. Journal of Guidance, Control and Dynamics 1988; 11(5):449-458. 